\title{
Integrated in vitro models for hepatic safety and metabolism: evaluation of a human Liver-Chip and liver spheroid
}

\author{
Alison J. Foster ${ }^{1}\left[\right.$ B Bhavik Chouhan ${ }^{2} \cdot$ Sophie L. Regan ${ }^{1} \cdot$ Helen Rollison $^{1} \cdot$ Sara Amberntsson $^{2}$. \\ Linda C. Andersson ${ }^{2} \cdot$ Abhishek Srivastava $^{1} \cdot$ Malin Darnell $^{2} \cdot$ Jonathan Cairns $^{3} \cdot$ Stanley E. Lazic ${ }^{3} \cdot$ Kyung-Jin Jang $^{4}$. \\ Debora B. Petropolis ${ }^{4} \cdot$ Konstantia Kodella ${ }^{4}$. Jonathan E. Rubins ${ }^{4} \cdot$ Dominic Williams $^{1} \cdot$ Geraldine A. Hamilton $^{4}$. \\ Lorna Ewart ${ }^{1} \cdot$ Paul Morgan ${ }^{1}$
}

Received: 19 November 2018 / Accepted: 4 March 2019 / Published online: 26 March 2019

(c) The Author(s) 2019

\begin{abstract}
Drug-induced liver injury remains a frequent reason for drug withdrawal. Accordingly, more predictive and translational models are required to assess human hepatotoxicity risk. This study presents a comprehensive evaluation of two promising models to assess mechanistic hepatotoxicity, microengineered Organ-Chips and 3D hepatic spheroids, which have enhanced liver phenotype, metabolic activity and stability in culture not attainable with conventional 2D models. Sensitivity of the models to two hepatotoxins, acetaminophen (APAP) and fialuridine (FIAU), was assessed across a range of cytotoxicity biomarkers (ATP, albumin, miR-122, $\alpha$-GST) as well as their metabolic functionality by quantifying APAP, FIAU and CYP probe substrate metabolites. APAP and FIAU produced dose- and time-dependent increases in miR-122 and $\alpha$-GST release as well as decreases in albumin secretion in both Liver-Chips and hepatic spheroids. Metabolic turnover of CYP probe substrates, APAP and FIAU, was maintained over the 10-day exposure period at concentrations where no cytotoxicity was detected and APAP turnover decreased at concentrations where cytotoxicity was detected. With APAP, the most sensitive biomarkers were albumin in the Liver-Chips $\left(\mathrm{EC}_{50} 5.6 \mathrm{mM}\right.$, day 1) and miR-122 and ATP in the liver spheroids (14-fold and $\mathrm{EC}_{50} 2.9 \mathrm{mM}$, respectively, day 3). With FIAU, the most sensitive biomarkers were albumin in the Liver-Chip ( $\mathrm{EC}_{50}$ $126 \mu \mathrm{M})$ and miR-122 (15-fold) in the liver spheroids, both on day 7. In conclusion, both models exhibited integrated toxicity and metabolism, and broadly similar sensitivity to the hepatotoxicants at relevant clinical concentrations, demonstrating the utility of these models for improved hepatotoxicity risk assessment.
\end{abstract}

Keywords DILI $\cdot$ Spheroid $\cdot$ Liver-chip $\cdot$ Hepatocyte $\cdot$ Hepatotoxicity $\cdot$ Metabolism

\section{Abbreviations}

$\alpha$-GST Alpha-glutathione-s-transferase

APAP Acetaminophen

2D Two-dimensional

3D Three-dimensional

CHPM Liver-Chip hepatocyte plating medium

Alison J. Foster and Bhavik Chouhan contributed equally to this work.

Electronic supplementary material The online version of this article (https://doi.org/10.1007/s00204-019-02427-4) contains supplementary material, which is available to authorized users.

Alison J. Foster

alison.foster2@astraeneca.com;

alison.foster2@astrazeneca.com

Extended author information available on the last page of the article

$\begin{array}{ll}\text { DILI } & \text { Drug-induced liver injury } \\ \text { ELISA } & \text { Enzyme-linked immunosorbent assay } \\ \text { FIAU } & \text { Fialuridine } \\ \text { HCM } & \text { Liver spheroid hepatocyte culture medium } \\ \text { HMM } & \text { Liver-Chip hepatocyte maintenance medium } \\ \text { HPM } & \text { Liver spheroid hepatocyte plating media } \\ \text { LSECGM } & \text { LSEC growth media } \\ \text { NPC } & \text { Non-parenchymal cells } \\ \text { PHH } & \text { Primary human hepatocytes } \\ \text { P450 } & \text { Cytochrome P450 } \\ \text { miR-122 } & \text { microRNA-122 } \\ \text { qPCR } & \text { Quantitative polymerase chain reaction } \\ \text { RT } & \text { Real time } \\ \text { ULA } & \text { Ultra-low adhesion } \\ \text { UPLC } & \text { Ultra-performance liquid chromatography }\end{array}$




\section{Introduction}

Drug-induced liver injury (DILI) is one of the most frequent reasons for withdrawal of approved drugs from the market, and represents a huge burden on the healthcare system, accounting for up to $50 \%$ of acute liver failure cases (Lee et al. 2016; Lee 2017). A conceptual framework for the mechanism of action of chemical toxins at the chemical, biochemical, cellular and clinical levels has been developed over the last decades through major advances in molecular pharmacology and toxicology. Using this framework, we are now able to design in vitro models that better reflect the in vivo processes that link drug metabolism and the formation of toxic metabolites to changes in cell function. An additional need is to build translational models that improve hepatotoxicity predictions for which more integrated in vitro models would contribute (Park et al. 2011; Williams et al. 2013).

Current in vitro test systems used by the pharmaceutical industry include simple liver-derived cell-based or sub-cellular models that are poorly predictive of toxicological potential over a diverse chemical space. Importantly, such models take no account of the mechanistic basis of human DILI or the environmental conditions under which it might occur. Also critically, there has been no concerted effort to harmonise current, emerging and novel test systems, or to develop strategies for their implementation, across the pharmaceutical industry (Williams et al. 2013). Consequently, too little is understood about how current test systems compare physiologically with human liver, what the critical signalling systems are and the mechanisms by which DILI occurs in man, to be able to produce more predictive test systems. Simultaneous combination of both metabolic and cytotoxic endpoints is required to gain an integrated understanding of how drug metabolism affects hepatocyte health (Bell et al. 2016, 2018; Sison-Young et al. 2017; Stachulski et al. 2013).

Two of the most promising areas of research for novel in vitro hepatic co-culture models are 3D spheroids/microtissues and flow-based microphysiological systems (MPS) (Esch et al. 2015; Ewart et al. 2018; Pridgeon et al. 2018; Whitman et al. 2016; Williams et al. 2013). Both have demonstrated superior enzyme expression or functionality of phase I and II, and transporter proteins over simple 2D cultures in extended culture periods (Bell et al. 2018; Tsamandouras et al. 2017). One of the main benefits of these novel models is that they allow maintenance of an in vivolike phenotype for longer periods versus 2D static cultures. For example, 3D hepatic spheroid/microtissue cultures show enhanced metabolic and toxicological phenotypes with superior sensitivity and specificity in detecting DILI compounds over 2D systems, in some cases with the cells from the same donor (Bell et al. 2016, 2018; Proctor et al. 2017). Additionally, complex models can include non-parenchymal cells (NPCs) necessary for stabilising chronic hepatocyte function, mimicing the permeability properties of the liver endothelium and modelling of further DILI mechanisms, such as involvement of the innate immune system and fibrosis (Bell et al. 2018; Li 2018; Vorrink et al. 2018). Moreover, towards replacing the use of animal-derived tissue or in vivo models, MPS can recapitulate the liver sinusoid (Bale et al. 2014; Ewart et al. 2018; Lee-Montiel et al. 2017). Of these systems, Organ-Chips have been designed to control the cellular microenvironment and to emulate the environment present within the liver in vivo (Du et al. 2017).

Here, we have evaluated the metabolic and toxicological phenotype of two novel in vitro models, using cryopreserved hepatocytes from the same human donor, 3D hepatic spheroids and a human Organ-Chip model (Liver-Chip). Metabolism and cytotoxic endpoints were assessed using two model hepatotoxic molecules, APAP and FIAU, over a 10-day culture period.

\section{Materials and methods}

Details of all materials, cell culture media formulations, a summary of the incubation and dosing conditions, and the sampling times can be found in Supplementary Methods.

\section{Liver-Chip preparation and cell seeding}

The Liver-Chip has been designed to recapitulate both the physical and multicellular architecture as well as tissue-tissue interfaces of the liver, while providing a dynamic microenvironment representing vascular perfusion. Liver-Chips, fabricated from polydimethylsiloxane (PDMS), contain two microchannels separated by a submillimeter-sized flexible, porous, PDMS membrane. A detailed description of the Liver-Chip can be found in Peel et al. (Peel et al. 2019).

Both Liver-Chip channels were coated with a proprietary mixture of extracellular matrix prior to overnight incubation in a humidified chamber at $37^{\circ} \mathrm{C}$. PHH were thawed, resuspended in hepatocyte plating media (CHPM), pelleted and resuspended prior to determination of cell number using trypan blue exclusion. Liver-Chips (top channel) were seeded with PHH $\left(3.5 \times 10^{6}\right.$ cell $\left./ \mathrm{ml}\right)$ and a monolayer was allowed to form over $24 \mathrm{~h}$ at $37{ }^{\circ} \mathrm{C}$ with $5 \% \mathrm{CO}_{2}$. The monolayer was then overlaid with ice-cold Matrigel ${ }^{\circledR}$ Matrix (200 $\mu \mathrm{l}$ $0.25 \mathrm{mg} / \mathrm{ml})$ in hepatocyte maintenance media (HMM) and the Liver-Chips were incubated overnight at $37^{\circ} \mathrm{C}$. The bottom channel was then seeded with LSECs $\left(3.4 \times 10^{6}\right.$ cells/ $\mathrm{ml}$ ) in LSEC iXCell endothelial growth medium (LSECGM). Liver-Chips were inverted and incubated at $37{ }^{\circ} \mathrm{C}$ for $2 \mathrm{~h}$ to allow LSEC attachment to the top surface of the bottom channel. Liver-Chips were then connected to a pneumatic pump and HMM or LSECGM flowed through the respective channels at $30 \mu \mathrm{l} / \mathrm{h}$. Compound treatment started 5 days 
after commencement of flow ( 7 days and 5 days post-seeding hepatocytes and LSECs to the Liver-Chips, respectively).

\section{Liver spheroid preparation and cell seeding}

PHH and NPCs were thawed separately in CM7000 media, pelleted and resuspended in hepatocyte plating medium (HPM). Cells were pelleted and resuspended a second time prior to cell number determination using trypan blue exclusion and seeding into 96-well ULA plates (final volume $100 \mu \mathrm{l} /$ well; 1350 hepatocytes/well and $150 \mathrm{NPCs} /$ well). Cells were allowed to aggregate under gravity over 4 days at $37{ }^{\circ} \mathrm{C}$ with $5 \% \mathrm{CO}_{2}$ prior to $50 \%$ medium change (repeated $2 \mathrm{x}$ ) with hepatocyte culture media (HCM). Drug exposure commenced after a further 3 days at $37{ }^{\circ} \mathrm{C}$ (7 days postseeding hepatocytes and NPCs).

\section{Toxicity study compound dosing and sampling}

DMSO (FIAU) or media (APAP) stock solutions were diluted in HCM or respective Liver-Chip media, HMM or LSEC dosing media, as detailed below.

\section{Liver-Chips}

Both channels were exposed to $0-100$ or $300 \mu \mathrm{M}$ FIAU, or $0-10 \mathrm{mM}$ APAP, (0.1\% DMSO v/v final) at $30 \mu \mathrm{l} / \mathrm{h}$ for 10 days. Media samples from the Liver-Chip outflow were collected for biomarker analysis and metabolite quantification/ identification over a $24-\mathrm{h}$ period on days $0-1$ (D1), days 2-3 (D3), days 6-7 (D7) and days 9-10 (D10). Dose solutions were replaced with freshly prepared dose solutions every 3 days. Duplicate chips were incubated with each drug concentration. All samples were stored at $-80^{\circ} \mathrm{C}$ prior to analysis. Adsorption of APAP and FIAU to PDMS was evaluated prior to commencement and no adsorption was observed.

\section{Liver spheroids}

Liver spheroids were exposed to $0-100$ or $300 \mu \mathrm{M}$ FIAU, or $0-10 \mathrm{mM}$ APAP, $(0.1 \%$ DMSO v/v final) for 10 days. Dose solutions were replenished by the replacement of $50 \%$ of the media in each well with freshly prepared dose solutions on days 4 and 7 . Twelve spheroids were incubated with each drug concentration. Two replicates were collected for biomarker analysis and metabolite identification/quantification on days $1,3,7$ and 10 by pooling $50 \mu \mathrm{l}$ media from 4 to 5 wells/concentration, to ensure sufficient sample was available for analysis of all endpoints, prior to storage at $-80^{\circ} \mathrm{C}$.

\section{CYP probe substrate dosing and sampling}

Pooled stocks containing 5 CYP probe substrates were prepared in acetonitrile or methanol prior to dilution in serum-free media to give the following final concentrations: phenacetin $30 \mu \mathrm{M}$ (CYP1A2); diclofenac $10 \mu \mathrm{M}$ (CYP2C9); S-mephenytoin $35 \mu \mathrm{M}$ (CYP2C19); bufuralol $5 \mu \mathrm{M}$ (CYP2D6) and midazolam $3 \mu \mathrm{M}$ (CYP3A4).

Table 1 Cytotoxicity assessment in Liver-Chips and liver spheroids

\begin{tabular}{|c|c|c|c|c|}
\hline \multicolumn{5}{|l|}{ A. APAP } \\
\hline \multirow[t]{2}{*}{$\mathrm{EC}_{50}(\mathrm{mM})$} & \multicolumn{4}{|l|}{ Day } \\
\hline & 1 & 3 & 7 & 10 \\
\hline \multicolumn{5}{|l|}{ Liver-chip } \\
\hline Albumin & $5.6 \pm 3.0$ & $3.1 \pm 1.4$ & $1.4 \pm 0.4$ & $1.5 \pm 0.4$ \\
\hline ATP & - & - & - & $2.4 \pm 0.2$ \\
\hline \multicolumn{5}{|c|}{ Liver spheroid } \\
\hline Albumin & $>10$ & $>10$ & $4.6 \pm 0.3$ & $2.4 \pm 0.5$ \\
\hline ATP & $>10$ & $2.9 \pm 1.0$ & $1.9 \pm 0.5$ & $1.7 \pm 0.6$ \\
\hline \multicolumn{5}{|l|}{ B. FIAU } \\
\hline \multirow[t]{2}{*}{$\mathrm{EC}_{50}(\mu \mathrm{M})$} & \multicolumn{4}{|l|}{ Day } \\
\hline & 1 & 3 & 7 & 10 \\
\hline \multicolumn{5}{|l|}{ Liver-chip } \\
\hline Albumin & $>100 / 300$ & $>100 / 300$ & $126 \pm 93$ & $48 \pm 57$ \\
\hline ATP & - & - & - & $77 \pm 57$ \\
\hline \multicolumn{5}{|c|}{ Liver spheroid } \\
\hline Albumin & $>100 / 300$ & $>100 / 300$ & $>100 / 300$ & $111 \pm 36$ \\
\hline ATP & $>100 / 300$ & $>100 / 300$ & $>100 / 300$ & $84 \pm 54$ \\
\hline
\end{tabular}

Effect of (A) APAP and (B) FIAU on albumin secretion and ATP depletion (Liver-Chip day 10 only). Data are mean $\mathrm{EC}_{50}$ values $\pm \mathrm{SD}_{2} n=3 / 4$ 


\section{APAP}

(A)

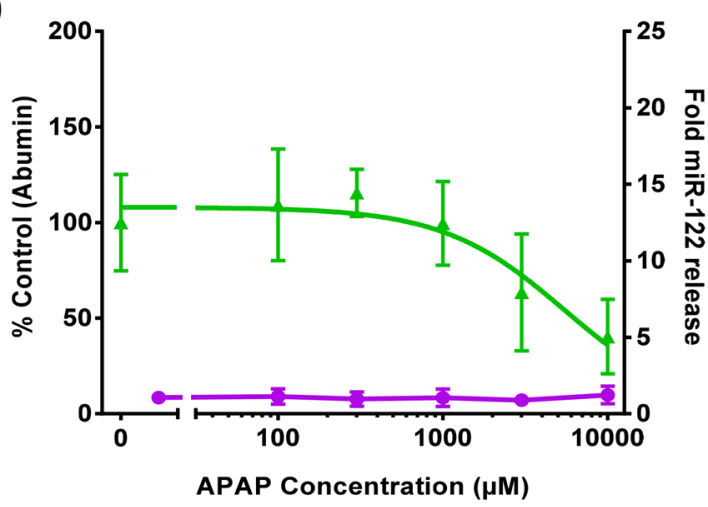

(B)

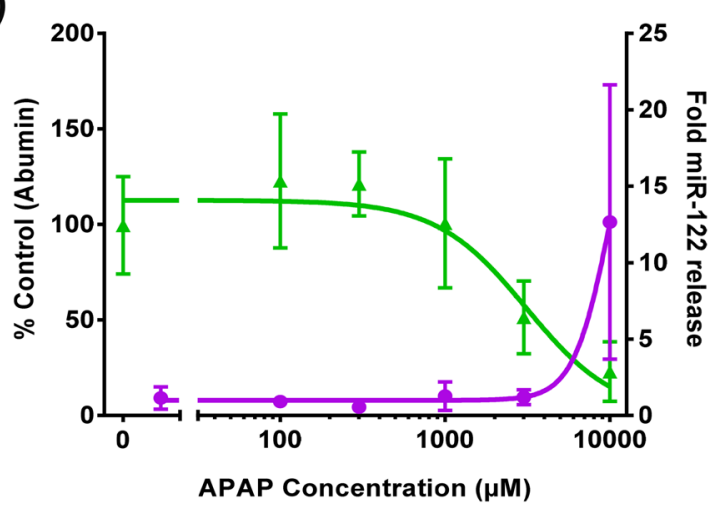

(C)

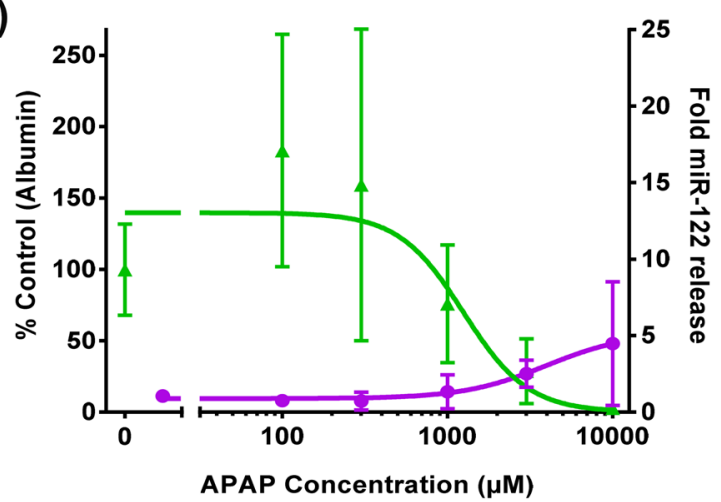

(D)

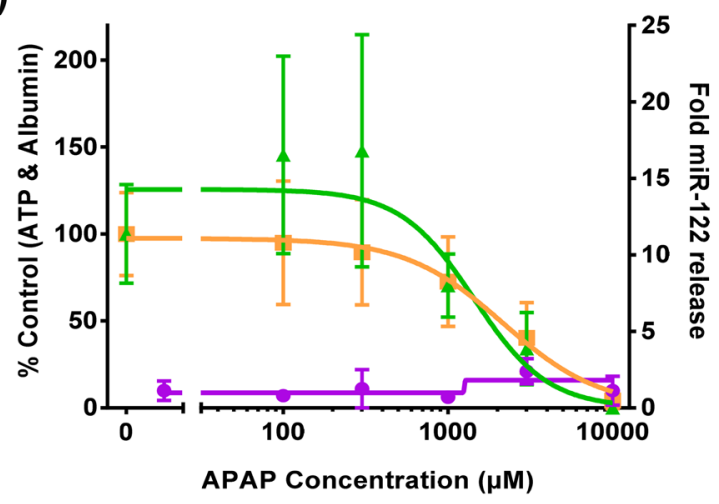

FIAU

(E)

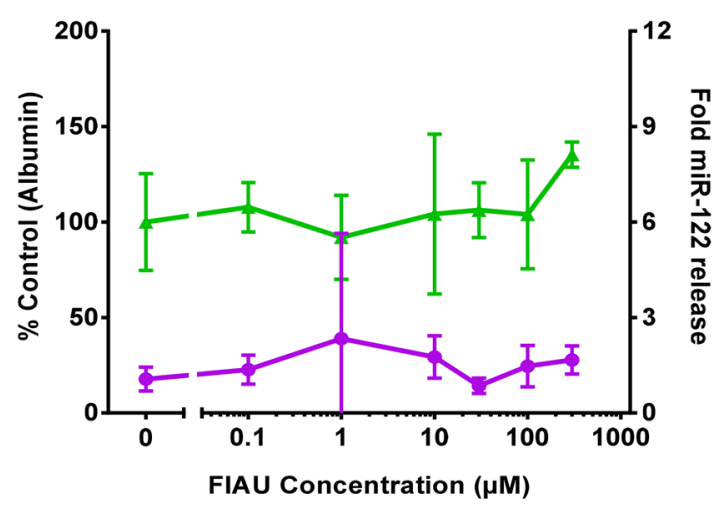

(F)

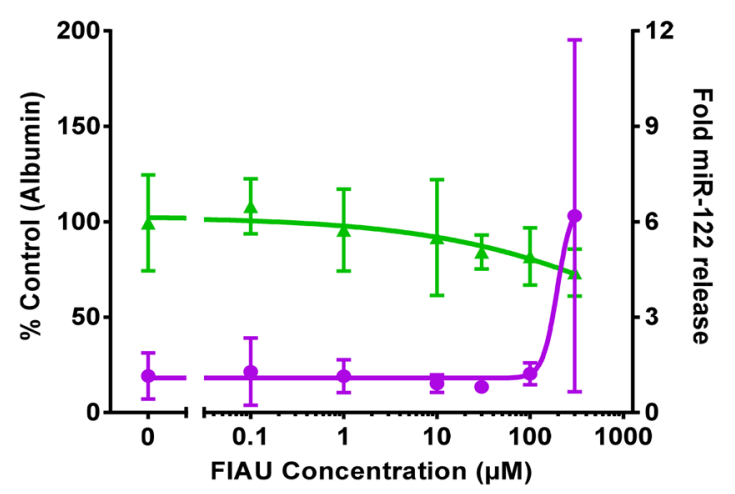

(G)

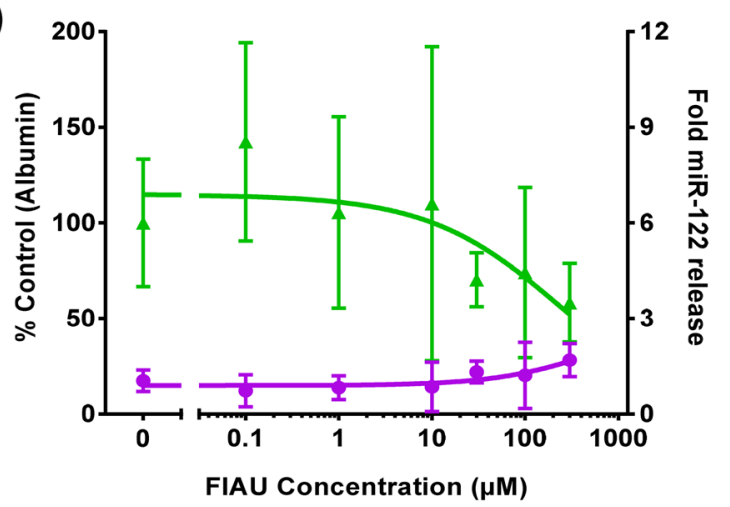

(H)

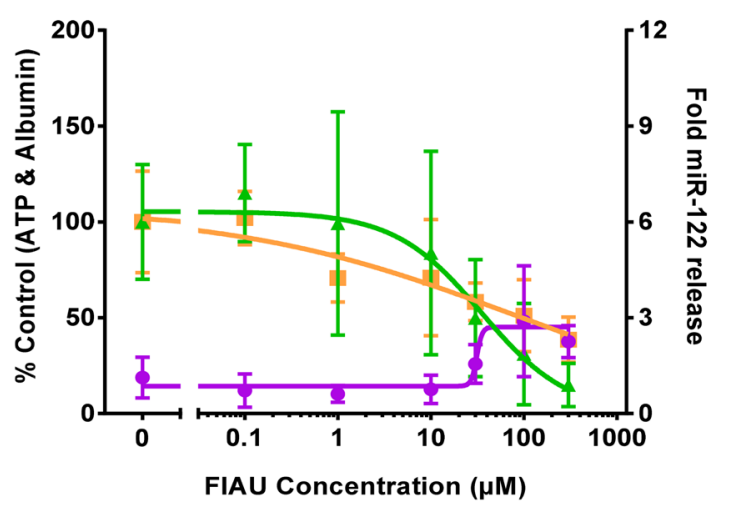


4Fig. 1 Evaluation of the toxicity of APAP and FIAU in Liver-Chips. Cells were exposed to $0-10,000 \mu \mathrm{M}$ APAP $(\mathbf{a}-\mathbf{d})$ or $0-100 / 300 \mu \mathrm{M}$ FIAU (e-h) for 10 days with 24-h sample collection on days (a, e) 0-1, (b, f) 2-3, (c, g) 6-7 and (d, h) 9-10 for (filled triangle) albumin and (filled circle) miR-122 release. On day 10, hepatocyte ATP levels and $\%$ viability of controls were determined (Filled Square). Data are mean $\pm \mathrm{SD},(n=3 / 4)$

\section{Liver-Chips}

A second pooled solution was prepared in DMSO prior to dilution in serum-free media to give the following final concentrations: chlorzoxazone $100 \mu \mathrm{M}$ (CYP2E1 probe substrate) and testosterone $200 \mu \mathrm{M}$ (CYP3A4 alternative probe). Liver-Chips were incubated with the respective substrate solutions at $30 \mu \mathrm{l} / \mathrm{h}$ for $24 \mathrm{~h}$ on days $0-1,2-3,6-7$ and 9-10. Samples were then collected, quenched 1:2 with acetonitrile containing $20 \mathrm{nM}$ verapamil and stored at $-80{ }^{\circ} \mathrm{C}$ prior to LC-MS analysis. Following sample collection, the media was replaced with HMM or LSECGM media and the cells were incubated under flow until the next time point.

\section{Liver spheroids}

Liver spheroids were exposed to the five pooled CYP probe substrates at the same final concentrations detailed above. Samples were collected following incubation for $24 \mathrm{~h}$ on days $0-1,3-4,7-8$ and 10-11, quenched 1:4 with acetonitrile containing $0.8 \%$ formic acid and $4 \mathrm{nM}$ 5,5-diethyl1,3-diphenyl-2-iminobarbituric acid, and stored at $-80{ }^{\circ} \mathrm{C}$.

\section{Cell viability assessment}

\section{Liver-Chips}

Hepatocytes were incubated with $100 \mu \mathrm{l}$ undiluted CellTiter-Glo ${ }^{\circledR}$ assay reagent for $1 \mathrm{~min}$ prior to agitation and

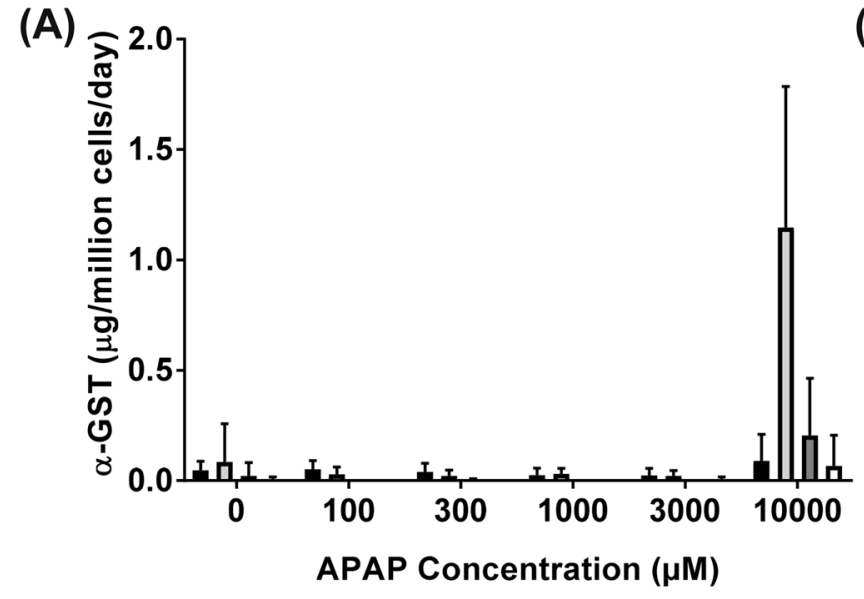

(C)

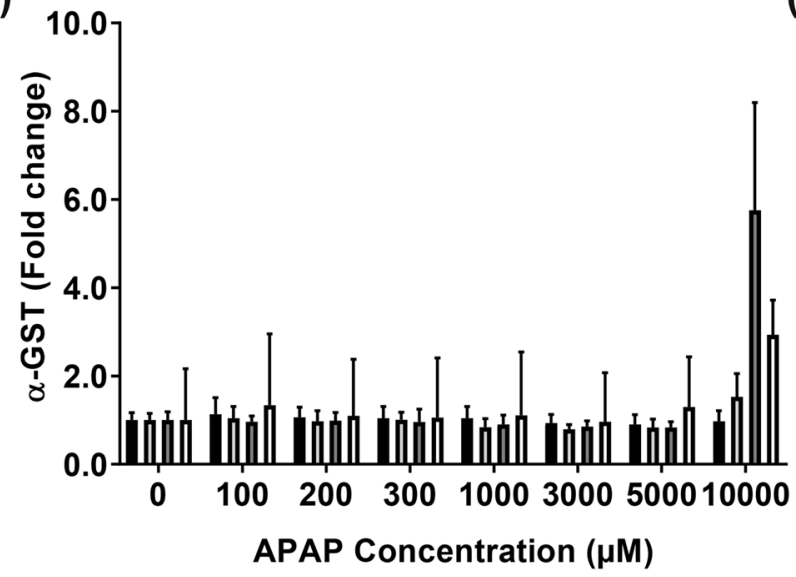

Fig. 2 Evaluation of $\alpha$-GST release by the Liver-Chip and liver spheroids following exposure to APAP or FIAU. Spheroids and LiverChips were exposed to $0-10,000 \mu \mathrm{M}$ APAP $(\mathbf{a}, \mathbf{c})$ or $0-100 / 300 \mu \mathrm{M}$ FIAU (b, d) for 10 days with samples collected on days 1, 3, 7 or
(B)

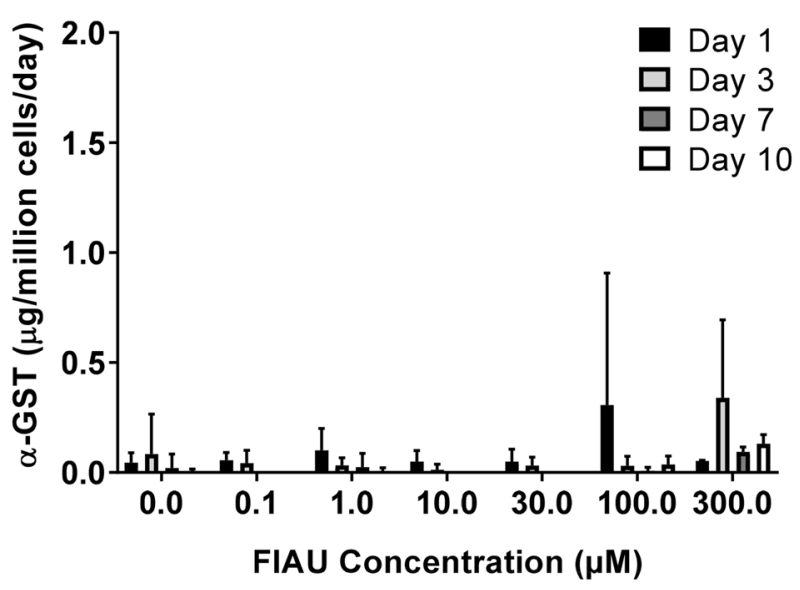

(D)

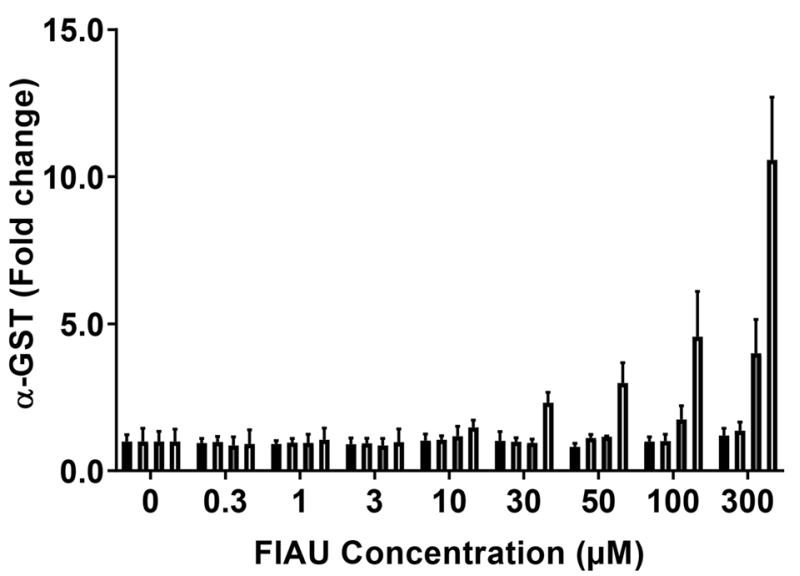

10 for the determination of release of $\alpha$-GST. Liver-Chip data (a, b) are expressed as $\mu \mathrm{g} / \mathrm{million}$ cells/day and spheroid data $(\mathbf{c}, \mathbf{d})$ as fold change with respect to the same day control. Data are mean $\pm \mathrm{SD}$, $(n=3 / 4)$ 


\section{APAP}

(A)

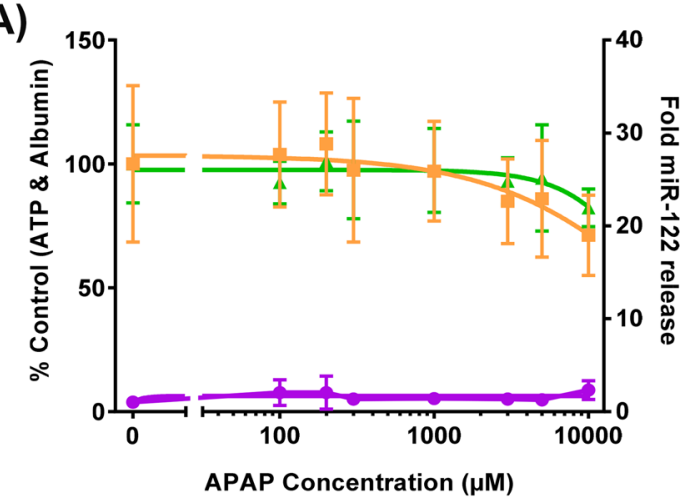

(B)

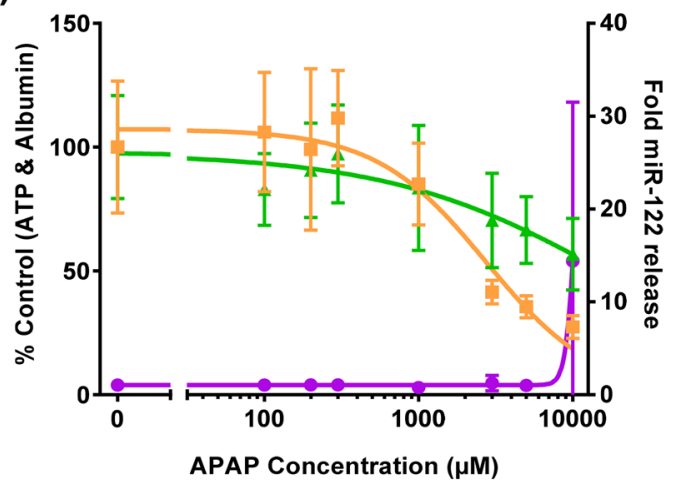

(C)

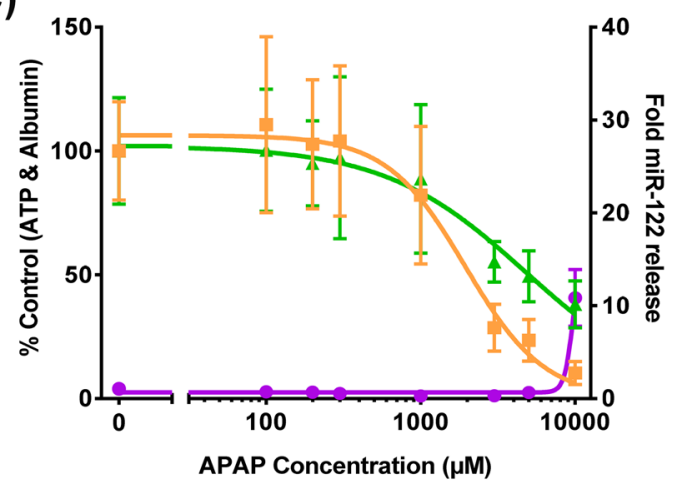

(D)

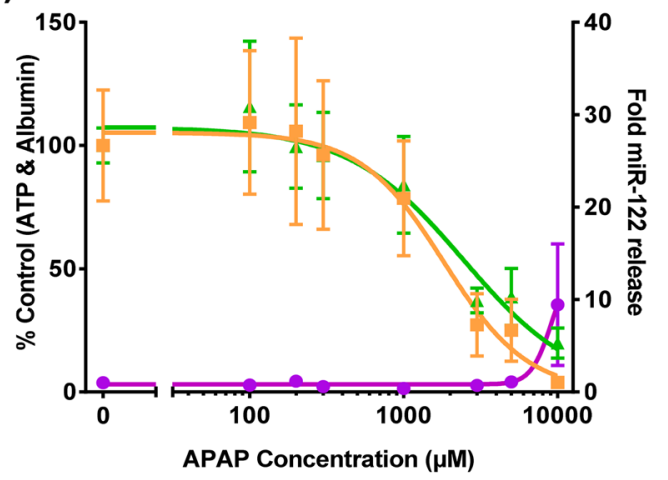

FIAU

(E)

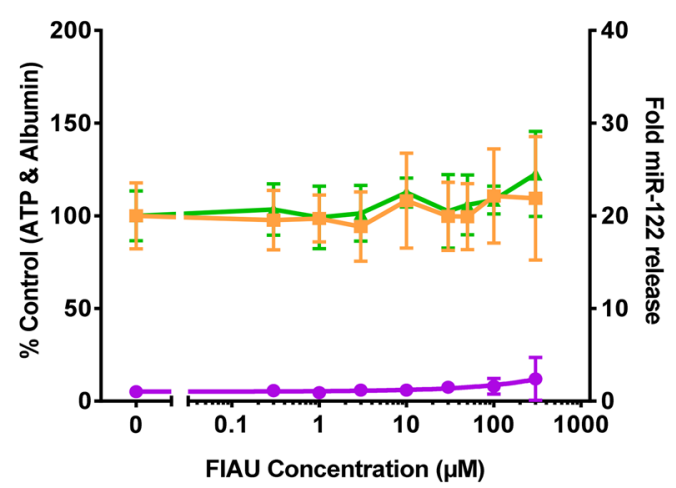

(F)

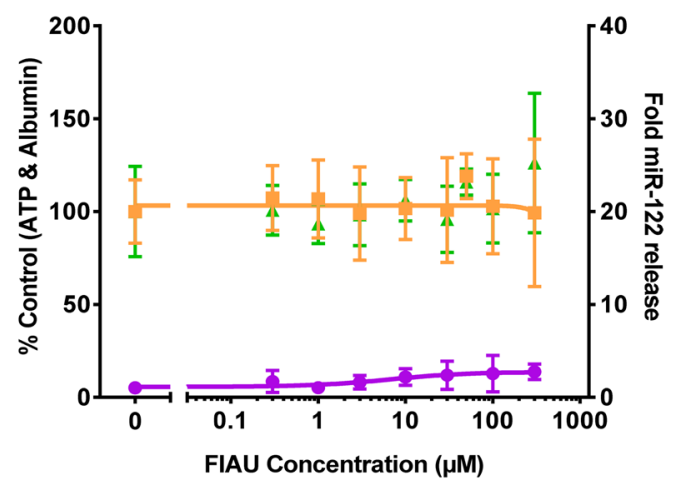

(G)

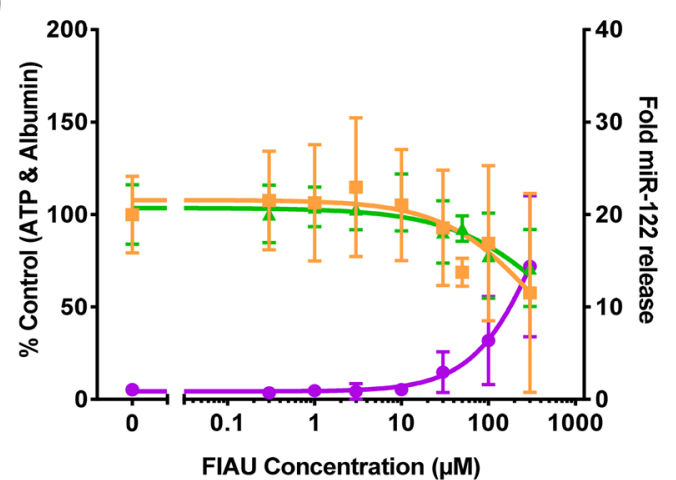

(H)

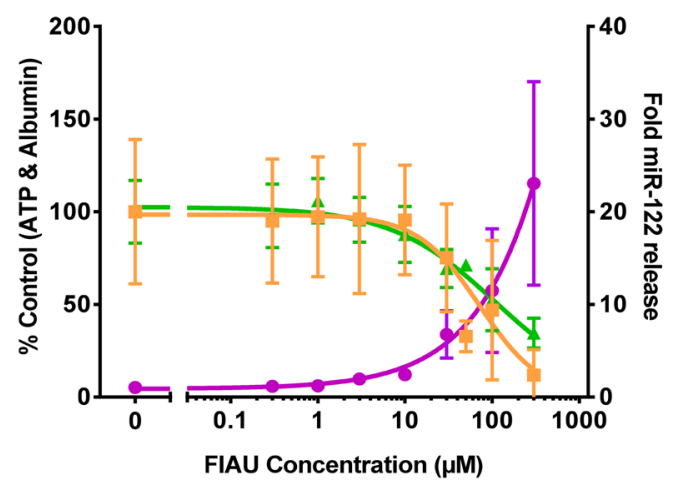


4 Fig. 3 Evaluation of the toxicity of APAP and FIAU in liver spheroids. Spheroids were exposed to $0-10,000 \mu \mathrm{M}$ APAP (a-d) or $0-100 / 300 \mu \mathrm{M}$ FIAU $(\mathbf{e}-\mathbf{h})$ for 10 days with samples collected for the determination of (filled triangle) albumin secretion and (filled circle) miR-122 release on days $1(\mathbf{a}, \mathbf{e}), 3(\mathbf{b}, \mathbf{f}), 7(\mathbf{c}, \mathbf{g})$ and $10(\mathbf{d}, \mathbf{h})$. Additionally, ATP levels and \% viability of controls were determined (Filled Square). Data are mean $\pm \mathrm{SD},(n=2 / 3)$

subsequent dilution with deionised water. The assay was then performed as per the manufacturer's protocol with luminescence determined on an Envison ${ }^{\mathrm{TM}}$ Multiplate Reader (Perkin Elmer, Waltham, MA, USA).

\section{Liver spheroids}

Cell viability was determined by the CellTiter-Glo® Assay following the manufacturer's protocol. Luminescence was determined on a SpectraMax iD3 Multi-Mode Microplate Reader (Molecular Devices, San Jose, CA, USA).

\section{Determination of a-GST activity}

Samples were analysed using the TecoMedical $\alpha$-GST ELISA assay. Briefly, samples $(50 \mu \mathrm{l})$ were transferred into microassay strips and $50 \mu \mathrm{l}$ diluent added. The ELISA was otherwise performed according to the manufacturer's instructions utilising an absorbance wavelength of $450 \mathrm{~nm}$.

\section{Determination of albumin levels}

Samples were analysed using the Bethyl Laboratories human serum albumin ELISA assay. Briefly, samples were diluted 1:20 (spheroids all days; Liver-Chip D7 and D10) or 1:50 (Liver-Chip D1 and D3) in sample diluent and $100 \mu$ diluted sample transferred into pre-coated assay plates. The ELISA was otherwise performed according to the manufacturer's instructions, apart from 1:90,000 dilution of the HRP Detection Antibody (Liver-Chips only).

\section{Determination of miR-122 levels}

miRNA extraction was performed using an miRNeasy 96 kit (Qiagen) following the manufacturer's instructions with minor modifications. Details of the extraction and RT PCR methodology are presented in Supplementary Methods.

\section{Determination of CYP probe substrate metabolite formation rates}

Formation rates of the probe substrate metabolites paracetamol (CYP1A2), 4-hydroxydiclofenac (CYP2C9), 4-hydroxymephenytoin (CYP2C19), 1-hydroxybufuralol (CYP2D6), 6-hydroxy-chlorzoxazone and 6-hydroxy-chlorzoxazoneglucuronide (CYP2E1), and 1-hydroxymidazolam and/or 6 $\beta$-hydroxytestosterone (CYP3A4) were determined by LC-MS as detailed in Supplementary Methods.

\section{APAP and FIAU metabolite identification/ quantification}

FIAU metabolite identification and quantification of APAP and its principle metabolites, APAP-glucuronide, -sulfate, - GSH and/or -cysteine conjugates, was performed by LC-MS as detailed in Supplementary Methods.

\section{Data analysis}

Cell viability and albumin data were normalized to vehicle control. Inhibition curves and $\mathrm{EC}_{50}$ estimates were generated by non-linear regression of log-transformed inhibitor concentrations (eight-point (spheroids) or six-point (LiverChip) serial dilutions including vehicle) for a sigmoidal dose-response using the four-parameter logistic equation in GraphPad Prism ${ }^{\mathrm{TM}}$ version 7.04 (GraphPad Software, La Jolla, CA, USA); bottom constrained to 0 .

$\alpha$-GST data were calculated as fold of control for liver spheroid samples and as $\mu \mathrm{g} / \mathrm{million}$ cells/day for Liver-Chip samples, as control levels were at or below the lower limit of detection $(1 \mathrm{ng} / \mathrm{ml})$ for some of the sampling days.

MiR-122- and cel-miR-39-measured threshold cycles were reported as Ct. Mean Ct of three-technical triplicates was used to calculate the relative release of miR-122 compared to cel-miR-39 spike-in $(\Delta \mathrm{Ct}) . \Delta \Delta \mathrm{Ct}$ was calculated for each sample relative to the mean of the vehicle controls at the matched timepoint, calculated as $2^{-\Delta \Delta \mathrm{Ct}}$ and reported as fold miR-122 release.

Masslynx ${ }^{\circledR}$ software version 4.1 and TargetLynx (Waters, U.K.) were used for analysis and processing of UPLC-MS/ MS data. Chromatographic peaks corresponding to the analyte were quantified using a calibration curve. The lower limit of quantification for each analyte can be found in Supplementary Table 11.

\section{Statistical analysis}

The technical variation between the two models was compared by calculating the residual variation (RV; the variation remaining after removing the effects of compound, day, and experiment) for each outcome. The RV (reported as \% total) was calculated for each outcome separately and for APAP and FIAU separately. The models were compared using a paired $t$ test. 


\section{Liver-Chip}

(A)

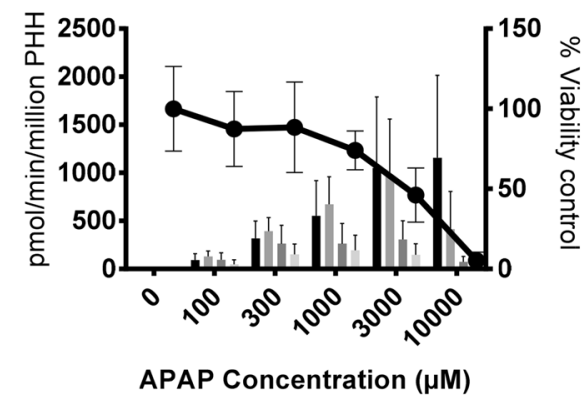

(C)

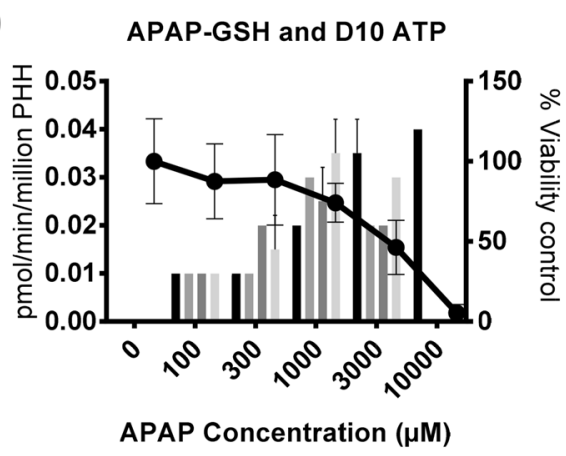

(B)

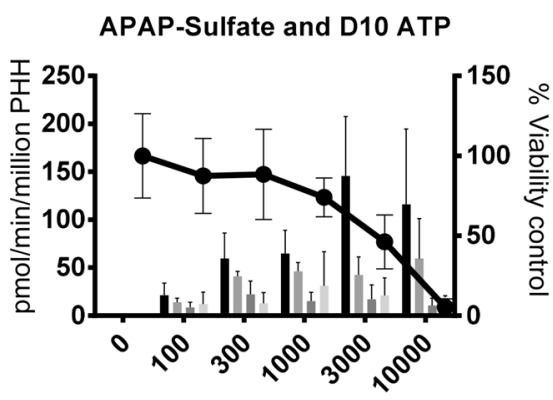

APAP Concentration $(\mu M)$

(D)

APAP-Cysteine and D10 ATP

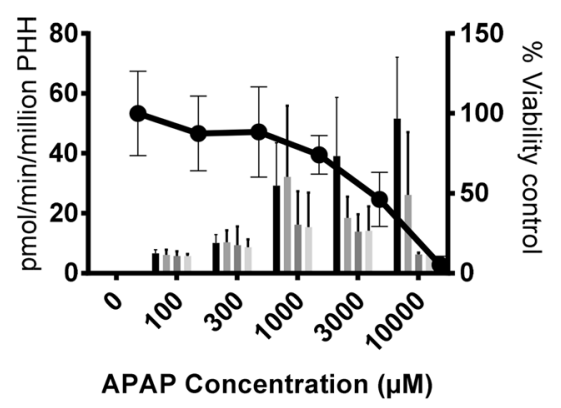

\section{Liver Spheroid}

(E)

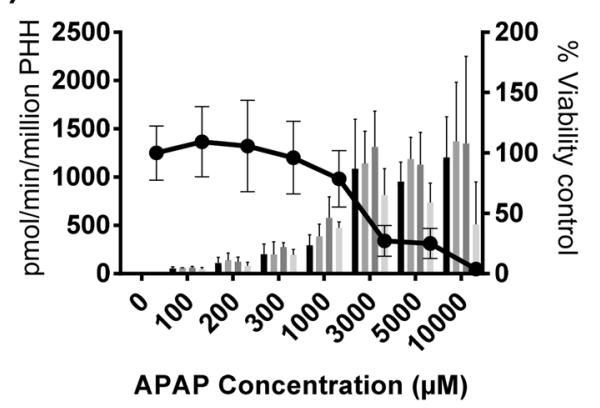

(F)

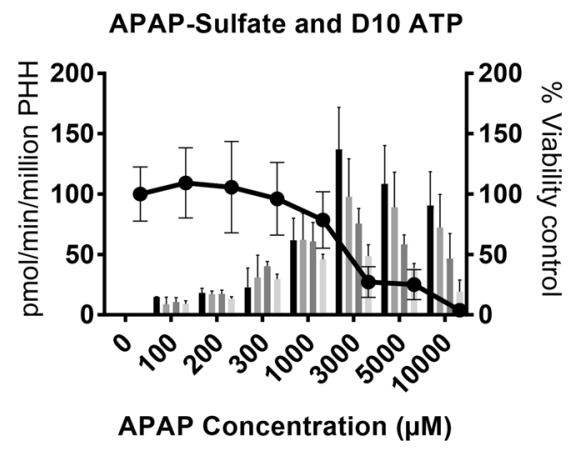

(G)

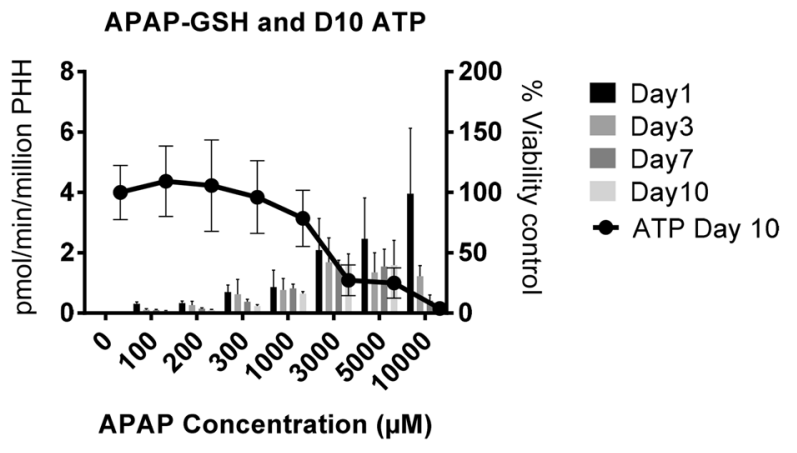


4Fig. 4 APAP Metabolic formation rates in Liver-Chips and liver spheroids. Liver-Chip hepatocyte channel samples were collected over days $0-1,2-3,6-7$ and 9-10 for the determination of APAPglucuronide, APAP-sulfate, APAP-GSH and APAP-cysteine metabolites (a-d) by LC-MS. Additionally, pooled spheroids samples were collected over days $0-1,0-3,4-7$ and 7-10 (e-g). Cell viability on day 10 was determined by ATP depletion and this profile is overlaid over the metabolism profiles. Data are mean $\pm \operatorname{SD}(n=3)$

\section{Results}

\section{Cytotoxicity and biomarker release profiles in Liver-Chips and liver spheroids}

Release of the hepatic biomarkers albumin (liver function), $\alpha$-GST and miR-122 (liver injury) was monitored on days 1, 3, 7 and 10 following the commencement of dosing. Additionally, ATP depletion (cell viability) was determined at all time points in liver spheroids and on day 10 in the LiverChips. A summary of the biomarker changes observed following exposure to APAP and FIAU can be found in Supplementary Table 12. Both models exhibited dose- and time-dependent cytotoxicity and decreased hepatocyte function following exposure to APAP and FIAU (Figs. 1, 2, 3; Table 1).

Control albumin levels in the Liver-Chip model ranged from 13 to $34 \mu \mathrm{g} / \mathrm{million}$ cells/day (Supplementary Fig. 1) which is equivalent to the albumin levels reported in the biochip OrganoPlateTM $(\sim 11-25 \mu \mathrm{g} / \mathrm{million}$ cells/day $)$ over a similar cultivation period (Jang et al. 2015). Sustained albumin production in perfused culture and a non-significant decline in albumin under dynamic conditions have been reported previously (Buesch et al. 2018; Vinci et al. 2011). However, due to the different units used, a direct comparison of the levels to those in the Liver-Chip model in this study was not possible.

Albumin was a sensitive biomarker of APAP cytotoxicity in Liver-Chips, with decreased secretion observed as early as 1 day after APAP dosing $\left(\mathrm{EC}_{50} 5.6 \pm 3.0 \mathrm{mM}\right)$. Additionally, peak increases in $\alpha$-GST and miR-122 release were observed at $10 \mathrm{mM}$ on day 3 (Figs. 1b, 2a). Although peak absolute values varied ( $\alpha$-GST, $0.2-1.7 \mu \mathrm{g} / \mathrm{million}$ cells/day; miR122, 7-30-fold), a similar trend was observed for both biomarkers in individual experiments. Moreover, dose-dependent decreases in hepatocyte albumin secretion and cell viability (ATP) exhibited a similar trend on day 10 (Fig. 1d; $\mathrm{EC}_{50} 1.5 \pm 0.4 \mathrm{mM}$ and $2.4 \pm 0.2 \mathrm{mM}$, respectively; Table 1). With FIAU, albumin was again the most sensitive biomarker in Liver-Chips. Dose- and time-dependent decreases were observed on days 7 and 10, although some interexperimental variations were observed $\left(\mathrm{EC}_{50} 126 \pm 93 \mu \mathrm{M}\right.$ and $48 \pm 57 \mu \mathrm{M}$ respectively; Fig. 1g, h; Table 1). Dose-dependent decreases in cell viability were also observed on day 10 , though the maximum decrease recorded at the top concentration was
$60 \%$ (300 $\mu \mathrm{M}$; Fig. 1h). With $\alpha$-GST, minimal release was observed on days $1-3(<0.4 \mu \mathrm{g} / \mathrm{million}$ cells/day; Fig. $2 \mathrm{~b})$ and only one to fivefold increases in miR-122 were observed on day 10 (Fig. 1h).

In liver spheroids, ATP depletion and miR-122 were early markers of APAP toxicity on day 3, with dose-dependent decreases in ATP levels at $>1 \mathrm{mM}\left(\mathrm{EC}_{50} 2.9 \pm 1.0 \mathrm{mM}\right)$ and 14-fold increases in miR-122 observed at $10 \mathrm{mM}$. Increased release of miR-122 was also observed on days 7 and 10 ( 10-fold; Fig. 3c, d) along with $\sim 6$ - and 3-fold increases, respectively in $\alpha$-GST (Fig. 2c). Moreover, similar profiles of decreases in albumin secretion and ATP were observed on day $10\left(\mathrm{EC}_{50}\right.$ values of $2.4 \pm 0.5 \mathrm{mM}$ and $1.7 \pm 0.6 \mathrm{mM}$ respectively; Fig. 3d; Table 1). MiR-122 was an early marker of toxicity for FIAU in liver spheroids. Marked increases in miR-122 release ( $\sim 15$-fold) were observed on day 7 with peak values of 23 -fold observed on day 10 (Fig. 3g, h). In contrast, marked dose-dependent decreases in albumin secretion and ATP levels were not observed until day $10\left(\mathrm{EC}_{50} 111 \pm 36 \mu \mathrm{M}\right.$ and $84 \pm 54 \mu \mathrm{M}$, respectively; Fig. 2h; Table 1). Increased $\alpha$-GST release was also observed on days 7 and 10 (Fig. 2d).

\section{APAP and FIAU metabolite identification}

Metabolite identification was performed on media samples from liver spheroids incubated with APAP or FIAU for 1, 3, 7 or 10 days. With APAP, four metabolites were identified (Scheme 1a), APAP-glucuronide (M1), APAP-cysteine (M2), APAP-GSH (M3) and APAP-sulfate (M4).

With FIAU, three accurate $\mathrm{m} / \mathrm{z}$ transformations were detected corresponding to proposed structures (Scheme 1b). Loss of 6.8930 (M1/M2) (suggesting des-Iodo-cysteine conjugate), loss of Iodine (M3) and two different glucuronide conjugates (M4/M5) appear at separate retention times (Supplementary Table 4, Supplementary Fig. 2). M1/M2, M3, M4 and M5 FIAU metabolites were also observed in LiverChip samples following incubation with $300 \mu \mathrm{M}$ FIAU for 3 days (Supplemental Fig. 3).

\section{APAP metabolite quantification in Liver-Chips and liver spheroids}

The concentration of APAP and its principle metabolites APAP-glucuronide, APAP-sulfate and APAP-GSH were quantified on days 1, 3, 7 and 10 following dosing (Fig. 4). For both models, turnover of APAP was observed at times and concentrations where cytotoxicity was not observed, whereas metabolite formation rates appeared to be impacted where cytotoxicity was present. APAP-glucuronide and APAP-sulfate were the major APAP metabolites detected (Fig. 4a, b, e, f).

The profiles of generated APAP metabolites were similar between Liver-Chip and liver spheroids apart from the 


\section{(A) APAP}

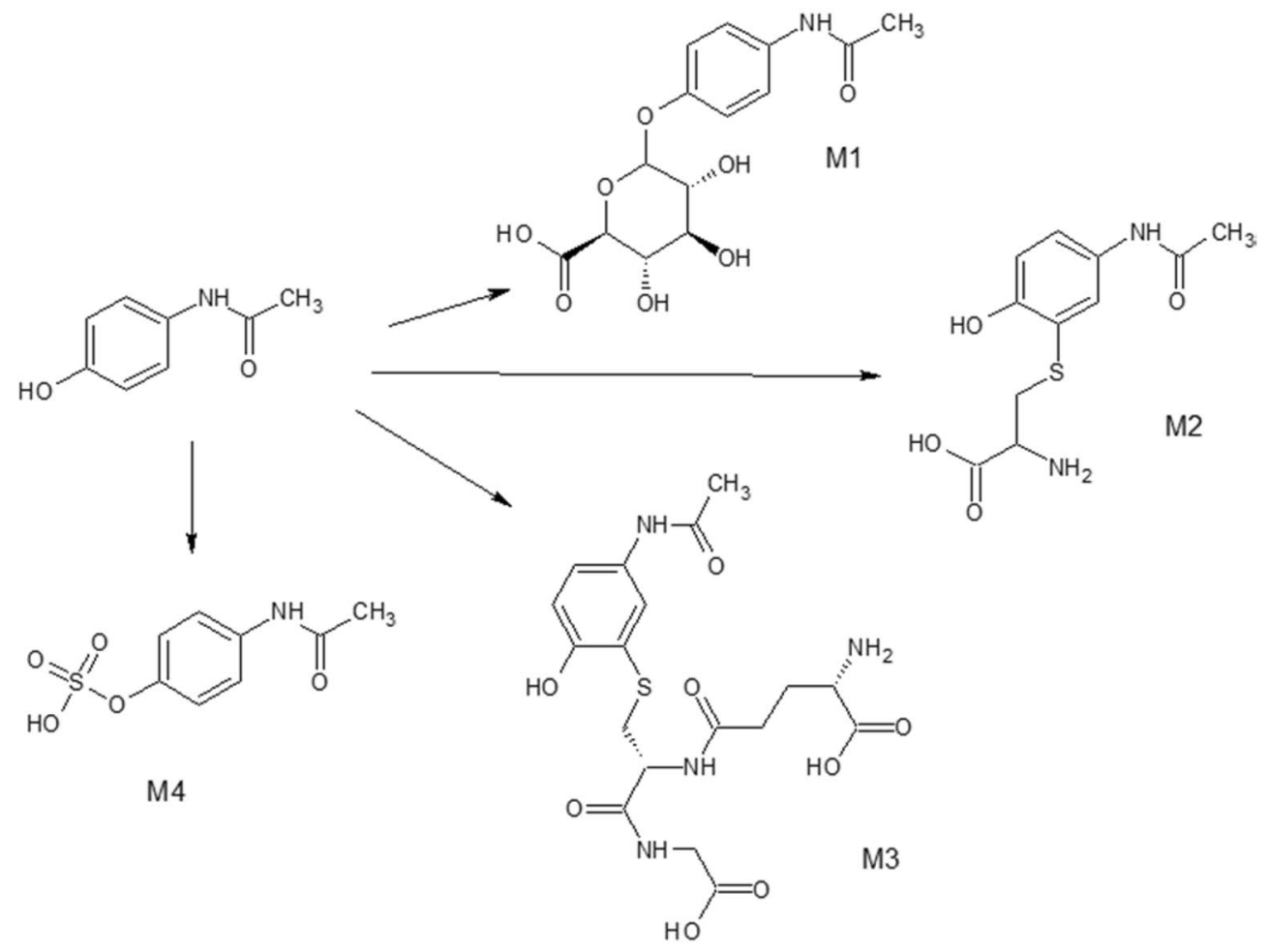

\section{(B) Fialuridine}

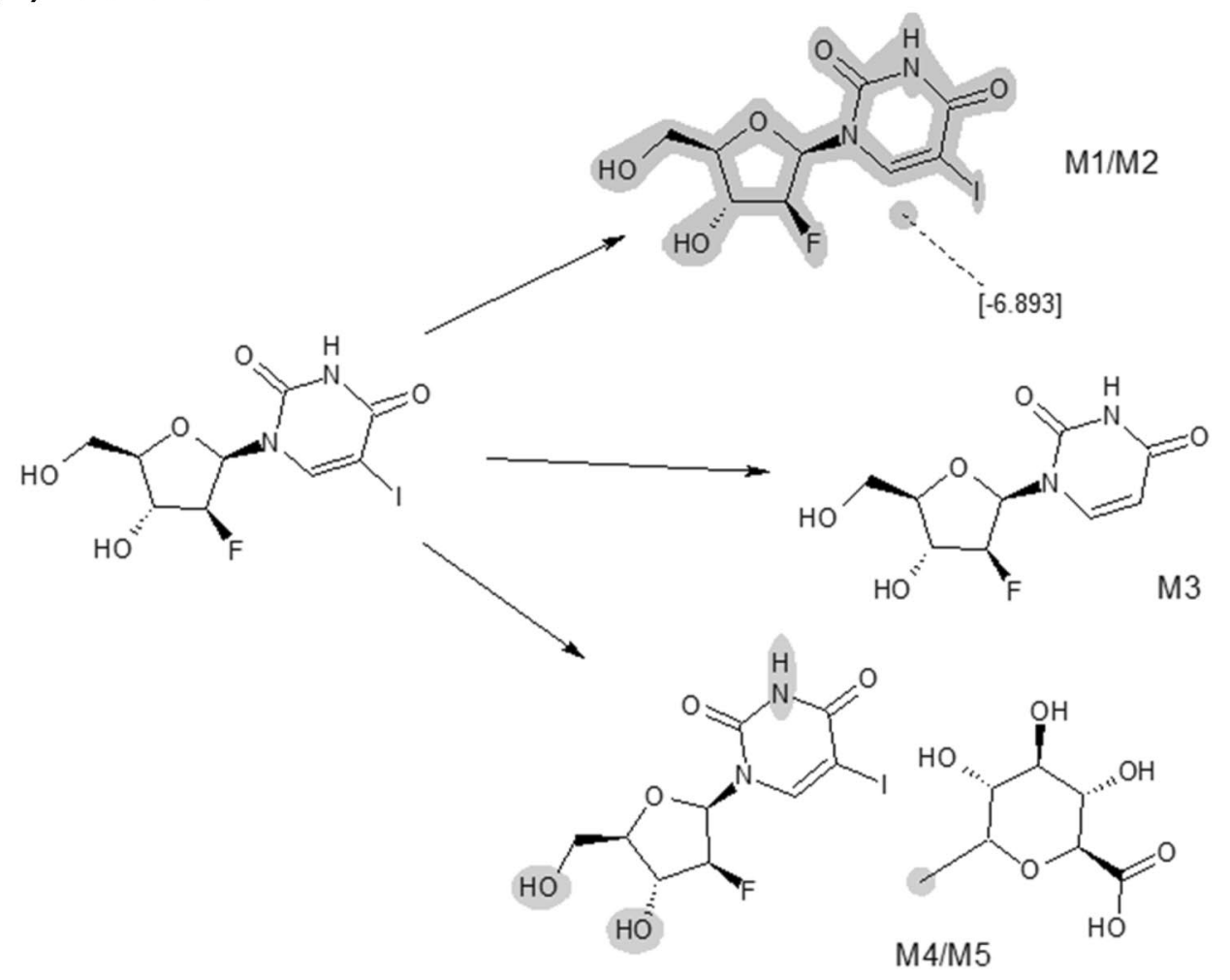


4Scheme 1 a Metabolites detected in the liver spheroid and LiverChip samples dosed with APAP: M1 (APAP-glucuronide), M2 (APAP-cysteine), M3 (APAP-GSH) and M4 (APAP-sulfate). b Proposed metabolites detected in the liver spheroid and Liver-Chip samples dosed with FIAU: M1/M2 (loss of 6.8930), M3 (loss of Iodine), and M4/M5 (glucuronide conjugate)

formation of the APAP-GSH conjugate (Fig. 4c, g). This difference could be explained by the high prevalence of cysteine conjugates in the Liver-Chip system (Fig. 4d): APAP-cysteine conjugate is a potential downstream metabolite of APAP-GSH conjugate (Vliegenthart et al. 2017) and so the low levels of APAP-GSH may be due to enhanced clearance of the metabolite in Liver-Chips. Also in the liver spheroid model, APAP-glucuronide and APAP-sulfate metabolites were detected at $\geq 3000 \mu \mathrm{M}$ despite low ATP levels being observed at the same concentrations on day 7 (Figs. 3c, 4e, f). This could be due to only $50 \%$ replacement of the media on days 4 and 7 to avoid 'loss' of the spheroid from the well, i.e. carry over of stable glucuronide and sulfate metabolites to the later time points, and/or possibly due to some residual enzyme activity prior to complete lysis of the cells.

\section{CYP activity profiling in Liver-Chips and liver spheroids}

CYP activity was maintained in both models over the duration of the study, although activity levels varied between and within experiments. Additionally, slightly lower CYP1A2 activity was observed in both models, and lower CYP2D6 activity was observed in liver spheroids, at later time points (Figs. 5, 6). In Liver-Chips relatively, low levels of $\mathrm{OH}$ midazolam were detected (Fig. 6d). An alternative CYP3A4 probe substrate, testosterone, was, therefore, evaluated. Much greater turnover of this substrate to its $6 \beta$-hydroxymetabolite was observed (Fig. 6f). For Liver-Chip CYP2E1 activity determination, formation rates for 6-hydroxy-chlorzoxazone-glucuronide (Fig. $6 \mathrm{~g}$ ) were measured as its precursor, 6-hydroxy-chlorzoxazone, was not detected.

\section{Technical variation}

Overall, no difference was observed in the technical variation of the Liver-Chip and liver spheroid models (Supplementary Fig. 4; paired $t$ test: $t_{6}=1.5, p=0.184$ ).

\section{Discussion}

DILI remains a major issue for the pharmaceutical industry, with failure to detect significant hepatotoxicity risk at nonclinical stages of the drug discovery/development pipeline having serious clinical repercussions with late stage and post-marketing attrition. Using in vitro cellular models to recapitulate hepatic function allows hazard identification and risk assessment of chemicals and drugs that may interfere

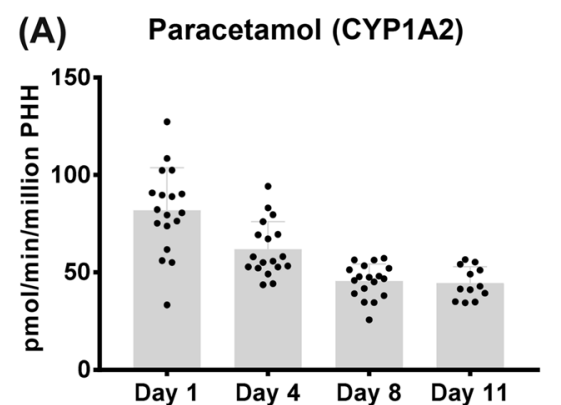

(D) OH-midazolam (CYP3A4)

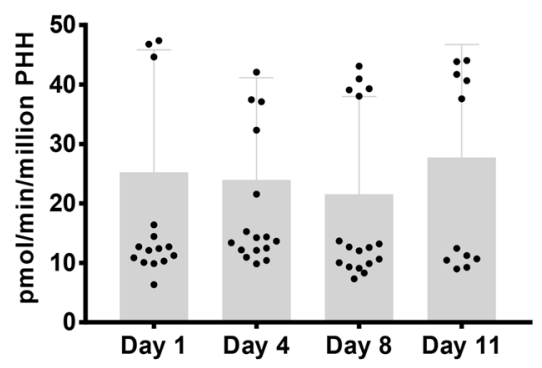

(B)

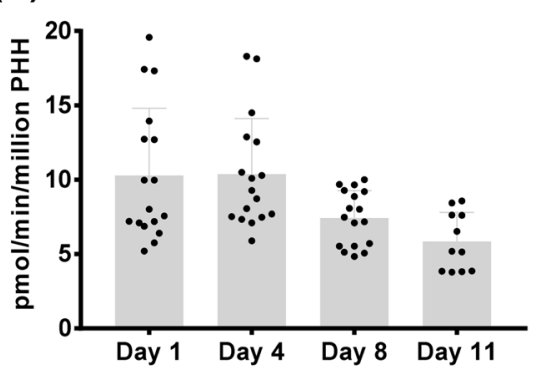

(E)

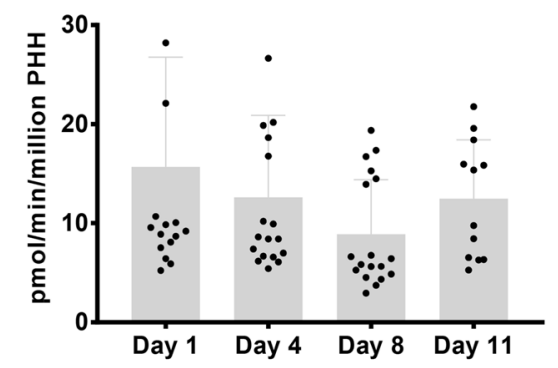

(C) OH-diclofenac (CYP2C9)

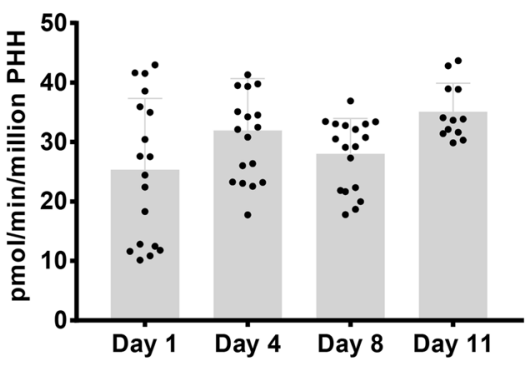

(F) OH-chlorzoxazone (CYP2E1)

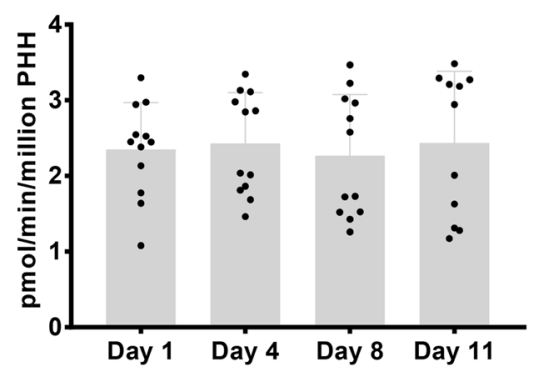

Fig. 5 Evaluation of CYP activity in liver spheroids. Spheroids were incubated with CYP probe substrates for $24 \mathrm{~h}$ on days $0-1,3-4,7-8$ and 10-11, and metabolites formed via CYP1A2 (a), CYP2D6 (b),
CYP2C9 (c), CYP3A4 (d), CYP2C19 (e) and CYP2E1 (f) were detected by LC-MS. Data are mean $\pm \mathrm{SD}(n=3,6$ spheroids/experiment) 
(A) Paracetamol (CYP1A2)

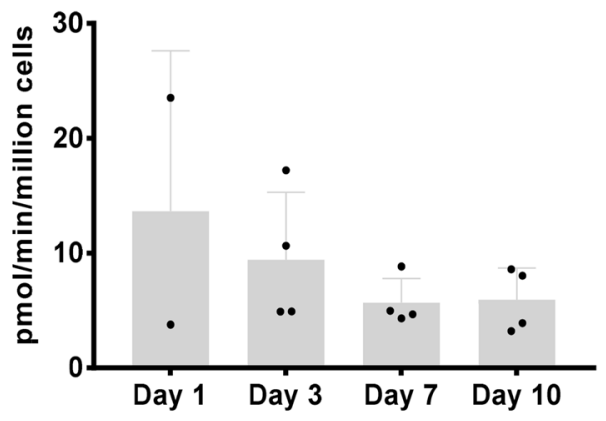

(C) OH-diclofenac (CYP2C9)

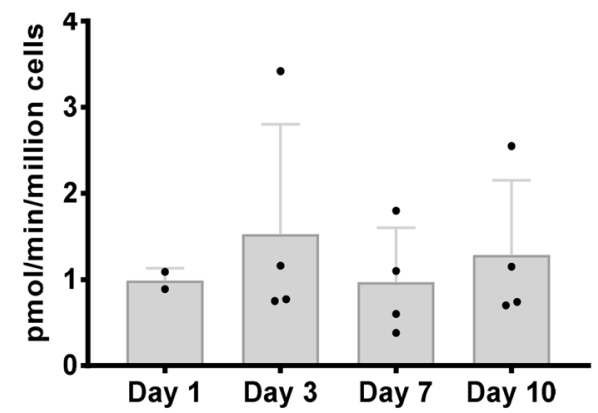

(E) OH-mephenytoin (CYP2C19)

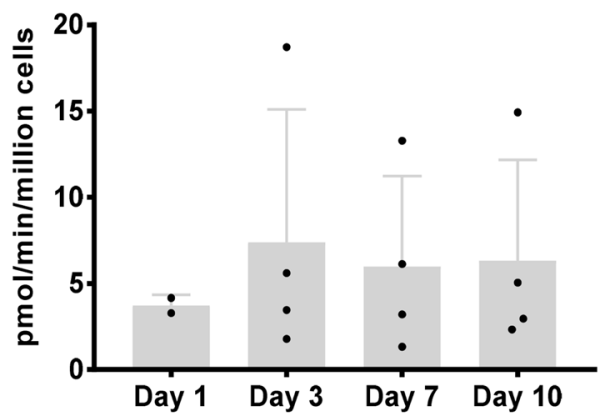

(G) OH-chlorzoxazone glucuronide (CYP2E1)

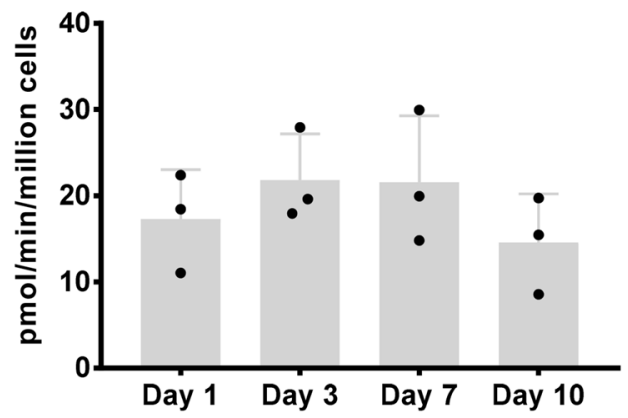

(B)

OH-bufuralol (CYP2D6)

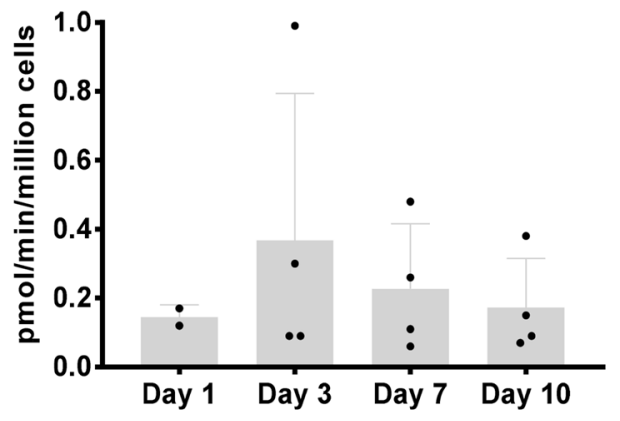

(D)

OH-midazolam (CYP3A4)

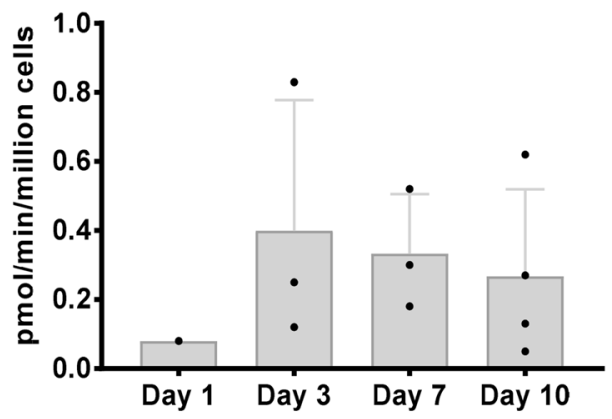

(F) 6 -hydroxytestosterone (CYP3A4)

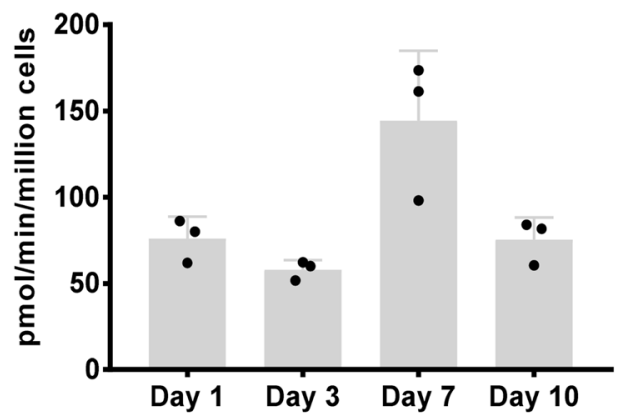


4Fig. 6 Evaluation of CYP activity in Liver-Chips. Liver-Chips were incubated with a CYP probe substrates for $24 \mathrm{~h}$ on days $0-1,2-3,6-7$ and 9-10, and metabolites formed via CYP1A2 (a), CYP2D6 (b), CYP2C9 (c), CYP3A4 (d, f), CYP2C19 (e), and CYP2E1 and phase II (g) detected by LC-MS. Pharmacokinetics was assessed under media flow conditions identical to the toxicity assessment protocol in this study, $30 \mu \mathrm{l} / \mathrm{h}$. Paracetamol and 1-hydroxy-midazolam have the potential to be further metabolised. Data are mean $\pm \mathrm{SD}(n=2$ in duplicates)

with homeostatic function resulting in adverse effects. The design of these models is crucial to ensure maintenance of the long-term hepatocyte and NPC phenotype; factors such as flow, cell-cell contact and co-culture each contribute to enable the in vitro hepatocyte to regain a more in vivo-like physiology. This study compared the effects of two model hepatotoxins, APAP and FIAU, on cryopreserved PHH from the same human donor in two in vitro models, hepatocyte/ NPC spheroid co-cultures and the Liver-Chip containing flow. Importantly, both models demonstrated functional drug metabolism and release of cellular stress and cytotoxicity biomarkers. This work has two novel aspects: first, we compared cells co-cultured in a static, scaffold-free 3D environment and those cultured in a more sinusoidal-like architecture with media flow; second, a seamless integration of cell stress/cytotoxicity endpoints with quantification of functional drug metabolism kinetics at equivalent concentrations and timepoints. In both models, NPCs are present, the hepatic spheroids containing 10\% mixed NPC and the Liver-Chips containing a 1:1 ratio with endothelial cells. The model hepatotoxins were selected because hepatocyte function is directly related to their mechanism of toxicity, for APAP this is reactive metabolite formation, glutathione depletion and covalent binding; for FIAU this is transportermediated uptake and integration into mitochondrial DNA (Honkoop et al. 1997). Biomarkers were selected based on two criteria. First, they needed to be sensitive enough when using only 1500 cells in the hepatic spheroid (Proctor et al. 2017). Second, circulating biomarkers (albumin, $\alpha$-GST and miR-122) were prioritized over destructive biomarkers (ATP) to enable the evaluation of chronic responses in the same incubation. Dose- and time-dependent release of $\alpha$-GST and relative expression of miR-122 into liver spheroid supernatants has been demonstrated previously in a proof of concept study in response to drug-induced cytotoxicity (Proctor et al. 2017).

For APAP, the most sensitive cytotoxicity biomarkers were albumin in the Liver-Chips, and ATP and miR-122 for the spheroids. ATP depletion was equivalent during FIAU administration. Similar sensitivity to APAP was observed in both models, but less sensitivity to FIAU as reported by Bell et al. $(2016,2018)$ in hepatic spheroids over a similar time course. Additionally, both models exhibited greater sensitivity to APAP than reported by Skardal et al. (2017) in liver constructs in a fluidic system. Marked decreases in albumin secretion and elevations of $\alpha$-GST release following exposure to $10 \mathrm{mM}$ APAP were not observed until 14 days and 10 days, respectively, in the liver construct model. Bell et al. (2018) reported that their proteomic dataset did not support an obvious correlation of the expression levels of various proteins known to play a role in FIAU mitochondrial uptake and its metabolism to the active triphosphate moiety, such as ENT1, TK2, and TMPK, with differences in sensitivity. However, differences in activity of these proteins or in the overall physiology of the mitochondria between 2D sandwich culture, 3D and Liver-Chips under flow conditions are possible and need to be tested to gain a further understanding of how these models can be used for particular DILI liabilities. Bell et al. (2018) also reported donor variability in 3D culture sensitivity to APAP and FIAU, particularly with APAP at $72 \mathrm{~h}$ and FIAU at 7 days, yet this disappeared with longer culture (14 days), likely due to gradual loss of CYP450 activities. We examined the same donor in both spheroids and Liver-Chips, and the sensitivity observed fits with the range of donor sensitivities previously reported (Bell et al. 2018). As CYP genes are highly variable between populations (Zhou et al. 2017) and previous studies have shown that genotypic differences can alter respective substrate pharmacokinetics (Vorrink et al. 2017), it is likely that larger scale investigations with donors from different ethnic backgrounds and with selected genotypes of interest will reveal more noticeable differences in drug sensitivities between donors in vitro (Bell et al. 2018).

Drug metabolites may initiate hepatotoxicity via multiple mechanisms, such as interfering with mitochondrial function or inhibiting the BSEP transporter (Dragovic et al. 2016). A recognised mechanism of hepatotoxicity is CYP450-mediated formation of reactive metabolites (Dragovic et al. 2016). Both APAP and FIAU undergo metabolism in human liver (Fein et al. 1985; Mazaleuskaya et al. 2015). At therapeutic doses, APAP undergoes direct Phase II detoxification via sulfation and glucuronidation, and is bioactivated to the reactive intermediate NAPQI via CYP450s. APAP-glucuronide has been reported as the most abundant human metabolite, followed by APAP-sulfate, whereas the APAP-GSH conjugate has been reported to be only a minor metabolite at therapeutic APAP dose levels (Critchley et al. 1986; Patel et al. 1992). This metabolic profile has been recapitulated in a cryopreserved hepatocyte suspension system, with approximately $85 \%$ of the drug appearing as the APAP-glucuronide conjugate at concentrations of $5 \mathrm{mM}$ (Riches et al. 2009). The metabolism of APAP presented here in the Liver-Chip and liver spheroid models is in line with the APAP metabolic profile reported in man in vivo and in human hepatocytes in vitro (Critchley et al. 1986; Patel et al. 1992; Riches et al. 2009). GSH conjugation of NAPQI (APAP-GSH) protects hepatocytes from reactive intermediates becoming covalently 
bound to hepatic protein and eventually hepatotoxicity (Laine et al. 2009; Mazaleuskaya et al. 2015). In both Liver-Chips and spheroids, GSH and cysteine conjugates of APAP were detected, indicating CYP450 activity, formation of NAPQI and preserved hepatic protection function via GSH conjugation in vitro. APAP-cysteine can either be formed directly from NAPQI (Streeter 1984) or by downstream metabolism via APAP-GSH (Vliegenthart et al. 2017). In vivo, NAPQI increases at toxic doses, which is captured in both models with significant increases in formation rates of APAP-GSH for spheroids and APAP-GSH and cysteine conjugates for Liver-Chips above toxic APAP concentrations $(>1 \mathrm{mM})$ (Mazaleuskaya et al. 2015; Pierce et al. 2002). For spheroids, APAP-GSH formation was absent for the highest concentrations between days 7 and 10, and no ATP was produced, suggesting cell death had prevented drug metabolism capability. A similar pattern was observed for APAP-cysteine in the Liver-Chips. APAP-glucuronide and APAP-sulfate formation is present during the culture period demonstrating phase II metabolism at comparable levels in both systems. Similar to in vivo, APAP-glucuronide is increased at supratherapeutic doses of APAP $(>0.1 \mathrm{mM})$ and saturated after highly toxic doses (> $3 \mathrm{mM}$ ) (Mazaleuskaya et al. 2015). APAP-glucuronide, APAP-sulfate and APAP-cysteine formations have been demonstrated previously in vitro in 2D culture cryopreserved human hepatocytes and HepaRG cells (Sjogren et al. 2014).

Specific enzyme activity of relevant P450s was assessed by measuring probe substrate metabolite formation, via CYP1A2, CYP2C19, CYP2C9, CYP2D6, CYP2E1 and CYP3A4. CYP3A4 and CYP2E1 are reported to be the major isoforms to bioactivate APAP to NAPQI at toxic doses, whereas other isoforms including CYP1A2, CYP2C19, CYP2C9 and CYP2D6 may also play a role at toxic and/or therapeutic doses (Chen et al. 1998; Laine et al. 2009; Raucy et al. 1989). Spheroid CYP activity levels were maintained during the study and demonstrated comparable activity levels to that observed in freshly thawed human hepatocytes, with the exception of CYP2E1 activity. The formation rates of both paracetamol (CYP1A2) and 4-hydroxy-diclofenac (CYP3A4) were observed to be in the range of that reported in freshly thawed cryopreserved human hepatocytes at $\mathrm{Km}$ (from nine donors) and $>1.4$-fold higher than H $\mu$ rel co-culture system for paracetamol. However, the CYP2E1 activity was observed to be 36 -fold lower than that observed in human hepatocytes from the same study ( $\mathrm{Li} \mathrm{2015).} \mathrm{The}$ formation rate of 1-hydroxy-midazolam was observed to be twofold higher than that reported in 16 hepatocyte donors at $\mathrm{Km}$ (calculated as Vmax/2). For 1-hydroxy-bufuralol, the formation rate was comparable to that reported in 10 donor pooled human hepatocytes (calculated as Vmax/2) and 14-fold higher than observed in the Hurel co-culture system. Furthermore, CYP2C19 activity, measured by the formation of 4-hydroxy-mephenytoin, was observed to be comparable to that reported in freshly thawed cryopreserved human hepatocytes (calculated as Vmax/2) in a different study (Brown et al. 2007). Liver-Chip CYP activity was also maintained throughout the study, but with relatively low levels of 1-hydroxy-midazolam formation (CYP3A4) (Fig. 6d). However, much greater levels of $6 \beta$-hydroxy-testosterone, formed from the alternative CYP3A4 probe substrate, testosterone, was observed (Fig. 6f). This indicates that metabolism of 1-hydroxy-midazolam to the glucuronide conjugate may be the reason for lower levels of 1-hydroxy-midazolam formation and not low CYP3A4 activity. Greater turnover of midazolam to its glucuronide in a dynamic Liver-Chip system versus a 2D-static system has been reported previously (Vivares et al. 2015). Here the amount 1-hydroxymidazolam had decreased due to turnover to its glucuronide. Phase II metabolism may also be responsible for the lower levels of paracetamol in the Liver-Chip compared to spheroids. Furthermore, for the determination of Liver-Chip CYP2E1 activity, formation rates for 6-hydroxy-chlorzoxazone-glucuronide were determined as its precursor 6-hydroxy-chlorzoxazone (Desiraju 1983) was not detected suggesting almost a complete conversion of this metabolite to the glucuronide.

FIAU is metabolised to FIAU glucuronide and des-iodoFIAU in humans, and the same metabolites were detected in spheroids and Liver-Chips (Bowsher et al. 1994; Fein et al. 1985). Additionally, a metabolite which has previously not been reported was observed in both systems suggesting the formation of electrophilic reactive species. This metabolite produced a mass of $[\mathrm{M}+\mathrm{H}]+366.0764 \mathrm{Da}$, corresponding to the cysteine conjugate of des-iodo-FIAU with the elemental composition C12H16FN3O7S. Further investigation of this proposed metabolite is required. Lewis et al. (Lewis et al. 1996) showed that parent FIAU decreased mitochondrial DNA replication in HepG2 cells. Thus, both FIAU and FIAU triphosphate could be involved in the mitochondrial hepatotoxicity observed in man.

In conclusion, both models demonstrated integrated drug metabolism and broadly similar sensitivity to the hepatotoxins at clinically relevant concentrations. The identification of FIAU as a hepatotoxicant in our models demonstrates that $3 \mathrm{D}$ co-culture and media flow/shear stress can result in the recapitulation of a more physiologically relevant in vitro system. Such an integration of metabolic bioactivation and markers of hepatotoxicity for APAP at relevant clinical concentrations demonstrates the utility of these models for improved hepatotoxicity risk assessment.

Acknowledgements We would like to thank Janey Ronxhi and Lian Leng for their excellent technical assistance.

Author contributions AJF, BC, SLR, HR, SA, LA and AS (study design, performed the experiments, biomarker analysis, data interpretation, drafting of manuscript); $\mathrm{MD}$ (data interpretation, drafting of manuscript); JC and SEL (formal analysis); DP, KK and JR 
(performed an experiment); KJJ (performed an experiment, review of manuscript); DW (study concept, design, data interpretation, drafting of manuscript); LE (study concept, data interpretation, critical review of manuscript); PM and GH (critical review of manuscript).

Funding No financial support.

Data availability All data generated or analysed during this study are included in this published article (and its supplementary files).

\section{Compliance with ethical standards}

Ethical statement The manuscript does not contain clinical studies or patient data.

Conflict of interest KJJ, GH, DP, KK and JR are employees of Emulate Inc.

Ethical approval This article does not contain any studies with human participants or animals performed by any of the authors.

Open Access This article is distributed under the terms of the Creative Commons Attribution 4.0 International License (http://creativeco mmons.org/licenses/by/4.0/), which permits unrestricted use, distribution, and reproduction in any medium, provided you give appropriate credit to the original author(s) and the source, provide a link to the Creative Commons license, and indicate if changes were made.

\section{References}

Bale SS, Vernetti L, Senutovitch N et al (2014) In vitro platforms for evaluating liver toxicity. Exp Biol Med (Maywood) 239(9):11801191. https://doi.org/10.1177/1535370214531872

Bell CC, Hendriks DF, Moro SM et al (2016) Characterization of primary human hepatocyte spheroids as a model system for druginduced liver injury, liver function and disease. Sci Rep 6:25187. https://doi.org/10.1038/srep25187

Bell CC, Dankers ACA, Lauschke VM et al (2018) Comparison of hepatic 2D sandwich cultures and 3D spheroids for long-term toxicity applications: a multicenter study. Toxicol Sci 162(2):655666. https://doi.org/10.1093/toxsci/kfx289

Bowsher RR, Compton JA, Kirkwood JA et al (1994) Sensitive and specific radioimmunoassay for fialuridine: initial assessment of pharmacokinetics after single oral doses to healthy volunteers. Antimicrob Agents Chemother 38(9):2134-2142

Brown HS, Griffin M, Houston JB (2007) Evaluation of cryopreserved human hepatocytes as an alternative in vitro system to microsomes for the prediction of metabolic clearance. Drug metabolism disposition 35(2):293-301

Buesch S, Schroeder J, Bunger M, D’Souza T, Stosik M (2018) A novel in vitro liver cell culture flow system allowing long-term metabolism and hepatotoxicity studies. Appl In Vitro Toxicol 4(3) https://doi.org/10.1089/aivt.2018.0009

Chen W, Koenigs LL, Thompson SJ et al (1998) Oxidation of acetaminophen to its toxic quinone imine and nontoxic catechol metabolites by baculovirus-expressed and purified human cytochromes P450 2E1 and 2A6. Chem Res Toxicol 11(4):295-301. https://doi. org/10.1021/tx9701687

Critchley JA, Nimmo GR, Gregson CA, Woolhouse NM, Prescott LF (1986) Inter-subject and ethnic differences in paracetamol metabolism. Br J Clin Pharmacol 22(6):649-657. https://doi. org/10.1111/j.1365-2125.1986.tb02953.x

Desiraju R, Renzi NL Jr, Nayak RK, NG, K-T (1983) Pharmacokinetics of chlorzoxazone in humans. J Pharm Sci 72(9):991-994. https:// doi.org/10.1002/jps.2600720905

Dragovic S, Vermeulen NP, Gerets HH et al (2016) Evidence-based selection of training compounds for use in the mechanismbased integrated prediction of drug-induced liver injury in man. Arch Toxicol 90(12):2979-3003. https://doi.org/10.1007/s0020 4-016-1845-1

Du Y, Li N, Yang H et al (2017) Mimicking liver sinusoidal structures and functions using a 3D-configured microfluidic chip. Lab Chip 17(5):782-794. https://doi.org/10.1039/c6lc01374k

Esch EW, Bahinski A, Huh D (2015) Organs-on-chips at the frontiers of drug discovery. Nat Rev Drug Discov 14(4):248-260. https:// doi.org/10.1038/nrd4539

Ewart L, Dehne E-M, Fabre K et al (2018) Application of microphysiological systems to enhance safety assessment in drug discovery. Annu Rev Pharmacol Toxicol 58(1):65-82. https://doi. org/10.1146/annurev-pharmtox-010617-052722

Fein G, Feinberg I, Insel TR et al (1985) Sleep mentation in the elderly. Psychophysiol 22(2):218-225

Honkoop P, Scholte HR, de Man RA, Schalm SW (1997) Mitochondrial injury. Lessons from the fialuridine trial. Drug Saf 17(1):1-7

Jang M, Neuzil P, Volk T, Manz A, Kleber A (2015) On-chip threedimensional cell culture in phaseguides improves hepatocyte functions in vitro. Biomicrofluidics 9(3):034113. https://doi. org/10.1063/1.4922863

Laine JE, Auriola S, Pasanen M, Juvonen RO (2009) Acetaminophen bioactivation by human cytochrome P450 enzymes and animal microsomes. Xenobiotica 39(1):11-21. https://doi. org/10.1080/00498250802512830

Lee WM (2017) Acetaminophen (APAP) hepatotoxicity-Isn't it time for APAP to go away? J Hepatol 67(6):1324-1331. https://doi. org/10.1016/j.jhep.2017.07.005

Lee SJ, Lee YJ, Park KK (2016) The pathogenesis of drug-induced liver injury. Expert Rev Gastroenterol Hepatol 10(10):11751185. https://doi.org/10.1080/17474124.2016.1196133

Lee-Montiel FT, George SM, Gough AH et al (2017) Control of oxygen tension recapitulates zone-specific functions in human liver microphysiology systems. Exp Biol Med (Maywood) 242(16):1617-1632. https://doi.org/10.1177/153537021770397 8

Lewis W, Levine ES, Griniuviene B et al (1996) Fialuridine and its metabolites inhibit DNA polymerase gamma at sites of multiple adjacent analog incorporation, decrease mtDNA abundance, and cause mitochondrial structural defects in cultured hepatoblasts. Proc Natl Acad Sci USA 93(8):3592-3597

Li AP (2015) Evaluation of adverse drug properties with cryopreserved human hepatocytes and the integrated discrete multiple organ coculture (IdMOCTM) system. Toxicol Res 31(2):137

Li XG, Vernetti SM, Gough L, Taylor AH, D.L (2018) A glass-based, continuously zonated and vascularized human liver acinus microphysiological system (vLAMPS) designed for experimental modeling of diseases and ADME/TOX. Lab Chip 18:2614-2631. https ://doi.org/10.1039/C8LC00418H

Mazaleuskaya LL, Sangkuhl K, Thorn CF, FitzGerald GA, Altman RB, Klein TE (2015) PharmGKB summary: pathways of acetaminophen metabolism at the therapeutic versus toxic doses. Pharmacogenet Genomics 25(8):416-426. https://doi.org/10.1097/ FPC.0000000000000150

Park BK, Boobis A, Clarke S et al (2011) Managing the challenge of chemically reactive metabolites in drug development. Nat Rev Drug Discov 10(4):292-306. https://doi.org/10.1038/nrd3408 
Patel M, Tang BK, Kalow W (1992) Variability of acetaminophen metabolism in Caucasians and Orientals. Pharmacogenetics 2(1):38-45

Peel S, Corrigan AM, Ehrhardt B et al (2019) Introducing an automated high content confocal imaging approach for organs-on-chips. Lab Chip 19(3):410-421. https://doi.org/10.1039/c81c00829a

Pierce RH, Franklin CC, Campbell JS et al (2002) Cell culture model for acetaminophen-induced hepatocyte death in vivo. Biochem Pharmacol 64(3):413-424

Pridgeon CS, Schlott C, Wong MW et al (2018) Innovative organotypic in vitro models for safety assessment: aligning with regulatory requirements and understanding models of the heart, skin, and liver as paradigms. Arch Toxicol 92(2):557-569. https://doi. org/10.1007/s00204-018-2152-9

Proctor WR, Foster AJ, Vogt J et al (2017) Utility of spherical human liver microtissues for prediction of clinical drug-induced liver injury. Arch Toxicol 91(8):2849-2863. https://doi.org/10.1007/ s00204-017-2002-1

Raucy JL, Lasker JM, Lieber CS, Black M (1989) Acetaminophen activation by human liver cytochromes P450IIE1 and P450IA2. Arch Biochem Biophys 271(2):270-283

Riches Z, Bloomer J, Patel A, Nolan A, Coughtrie M (2009) Assessment of cryopreserved human hepatocytes as a model system to investigate sulfation and glucuronidation and to evaluate inhibitors of drug conjugation. Xenobiotica 39(5):374-381. https:// doi.org/10.1080/00498250902763440

Sison-Young RL, Lauschke VM, Johann E et al (2017) A multicenter assessment of single-cell models aligned to standard measures of cell health for prediction of acute hepatotoxicity. Arch Toxicol 91(3):1385-1400. https://doi.org/10.1007/s0020 4-016-1745-4

Sjogren AK, Liljevald M, Glinghammar B et al (2014) Critical differences in toxicity mechanisms in induced pluripotent stem cellderived hepatocytes, hepatic cell lines and primary hepatocytes. Arch Toxicol 88(7):1427-1437. https://doi.org/10.1007/s0020 4-014-1265-z

Skardal A, Murphy SV, Devarasetty M et al (2017) Multi-tissue interactions in an integrated three-tissue organ-on-a-chip platform. Sci Rep 7(1) https://doi.org/10.1038/s41598-017-08879-x

Stachulski AV, Baillie TA, Park BK et al (2013) The generation, detection, and effects of reactive drug metabolites. Med Res Rev 33(5):985-1080. https://doi.org/10.1002/med.21273

Streeter A, Dahlin, DC, Nelson, SD, Baillie, TA (1984) < Streeter et al 1984 Chem Biol Int The covalent binding of acetaminophen to protein. Evidence for cysteine residues as major sites of arylation in vitro.pdf $>$. Chem Biol Interact 48:349-366
Tsamandouras N, Kostrzewski T, Stokes CL, Griffith LG, Hughes DJ, Cirit M (2017) Quantitative assessment of population variability in hepatic drug metabolism using a perfused three-dimensional human liver microphysiological system. J Pharmacol Exp Ther 360(1):95-105. https://doi.org/10.1124/jpet.116.237495

Vinci B, Duret c, Klieber S et al (2011) Modular bioreactor for primary human hepatocyte culture: Medium flow stimulates expression and activity of detoxification genes. Biotechnol J 6(5):554-564. https://doi.org/10.1002/biot.201000326

Vivares A, Salle-Lefort S, Arabeyre-Fabre C et al (2015) Morphological behaviour and metabolic capacity of cryopreserved human primary hepatocytes cultivated in a perfused multiwell device. Xenobiotica 45(1):29-44. https://doi.org/10.3109/00498 254.2014.944612

Vliegenthart A, Kimmitt RA, Seymour JH et al (2017) Circulating acetaminophen metabolites are toxicokinetic biomarkers of acute liver injury. Clin Pharmacol Ther 101(4):531-540. https://doi. org/10.1002/cpt.541

Vorrink SU, Ullah S, Schmidt S et al (2017) Endogenous and xenobiotic metabolic stability of primary human hepatocytes in longterm 3D spheroid cultures revealed by a combination of targeted and untargeted metabolomics. FASEB J 31(6):2696-2708. https ://doi.org/10.1096/fj.201601375R

Vorrink SU, Zhou Y, Ingelman-Sundberg M, Lauschke VM (2018) Prediction of drug-induced hepatotoxicity using long-term stable primary hepatic 3D spheroid cultures in chemically defined conditions. Toxicol Sci 163(2):655-665. https://doi.org/10.1093/ toxsci/kfy058

Whitman NA, McIntosh JC, Penley JB, Lockett MR (2016) Microfabricated devices for studying the metabolism and cytotoxicity of drug candidates. Curr Pharm Biotechnol 17(9):755-771

Williams DP, Shipley R, Ellis MJ et al (2013) Novel in vitro and mathematical models for the prediction of chemical toxicity. Toxicol Res (Camb) 2(1):40-59. https://doi.org/10.1039/c2tx20031g

Zhou Y, Ingelman-Sundberg M, Lauschke VM (2017) Worldwide distribution of cytochrome $\mathrm{P} 450$ alleles: a meta-analysis of population-scale sequencing projects. Clin Pharmacol Ther 102(4):688700. https://doi.org/10.1002/cpt.690

Publisher's Note Springer Nature remains neutral with regard to jurisdictional claims in published maps and institutional affiliations.

\section{Affiliations}

\section{Alison J. Foster ${ }^{1}(1) \cdot$ Bhavik Chouhan ${ }^{2} \cdot$ Sophie L. Regan ${ }^{1} \cdot$ Helen Rollison $^{1} \cdot$ Sara Amberntsson $^{2}$. Linda C. Andersson ${ }^{2} \cdot$ Abhishek Srivastava $^{1} \cdot$ Malin Darnell $^{2}$. Jonathan Cairns ${ }^{3} \cdot$ Stanley E. Lazic ${ }^{3} \cdot$ Kyung-Jin Jang $^{4}$. Debora B. Petropolis ${ }^{4} \cdot$ Konstantia Kodella ${ }^{4}$. Jonathan E. Rubins ${ }^{4}$. Dominic Williams ${ }^{1}$. Geraldine A. Hamilton ${ }^{4}$. Lorna Ewart ${ }^{1} \cdot$ Paul Morgan ${ }^{1}$}

Bhavik Chouhan

Bhavik.Chouhan@astrazeneca.com

Sophie L. Regan

sophie.regan@astrazeneca.com

Helen Rollison

Helen.Rollison@astrazeneca.com

Sara Amberntsson

Sara.Amberntsson@astrazeneca.com
Linda C. Andersson

Linda.C.Andersson@astrazeneca.com

Abhishek Srivastava

Abhishek.Srivastava@astrazeneca.com

Malin Darnell

Malin.Darnell@astrazeneca.com

Jonathan Cairns

jonathan.cairns@astrazeneca.com 
Stanley E. Lazic

stanley.lazic@astrazeneca.com

Kyung-Jin Jang

kj.jang@emulatebio.com

Debora B. Petropolis

debora.petropolis@emulatebio.com

Konstantia Kodella

konstantia.kodella@emulatebio.com

Jonathan E. Rubins

jonathan.rubins@gmail.com

Dominic Williams

Dominic.P.Williams@astrazeneca.com

Geraldine A. Hamilton

geraldine.hamilton@emulatebio.com
Lorna Ewart

Lorna.Ewart@astrazeneca.com

Paul Morgan

Paul.Morgan@astrazeneca.com

1 Drug Safety and Metabolism, AstraZeneca IMED Biotech Unit, Cambridge CB4 0WG, UK

2 Drug Safety and Metabolism, AstraZeneca IMED Biotech Unit, Gothenburg 431 83, Sweden

3 Discovery Sciences, AstraZeneca IMED Biotech Unit, Cambridge, UK

4 Emulate, Inc, 27, Drydock Ave, 02210 Boston, MA, US 